\title{
An axiomatic analysis of concordance-discordance relations
}

\author{
Denis Bouyssou ${ }^{*, a, b}$, Marc Pirlot $^{\mathrm{c}}$ \\ ${ }^{a}$ CNRS-LAMSADE, Place du Maréchal de Lattre de Tassigny, F-75775 Paris Cedex 16, France \\ ${ }^{b}$ Université Paris Dauphine, UMR7024, Place du Maréchal de Lattre de Tassigny, F-75775 \\ Paris Cedex 16, France \\ ${ }^{c}$ Faculté Polytechnique de Mons, 9, rue de Houdain, B-7000 Mons, Belgium
}

\begin{abstract}
Outranking methods propose an original way to build a preference relation between alternatives evaluated on several attributes that has a definite ordinal flavor. Indeed, most of them appeal the concordance / non-discordance principle that leads to declaring that an alternative is "superior" to another, if the coalition of attributes supporting this proposition is "sufficiently important" (concordance condition) and if there is no attribute that "strongly rejects" it (non-discordance condition). Such a way of comparing alternatives is rather natural. However, it is well known that it may produce binary relations that do not possess any remarkable property of transitivity or completeness. This explains why the axiomatic foundations of outranking methods have not been much investigated, which is often seen as one of their important weaknesses. This paper uses conjoint measurement techniques to obtain an axiomatic characterization of preference relations that can be obtained on the basis of the concordance / non-discordance principle. It emphasizes their main distinctive feature, i.e., their very crude way to distinguish various levels of preference differences on each attribute. We focus on outranking methods, such as ELECTRE I, that produce a reflexive relation, interpreted as an "at least as good as" preference relation. The results in this paper may be seen as an attempt to give such outranking methods a sound axiomatic foundation based on conjoint measurement.
\end{abstract}

Key words: Multiple criteria analysis, Concordance, Discordance, Outranking methods, Conjoint measurement, Nontransitive preferences

\footnotetext{
* Corresponding author

Email addresses: bouyssou@lamsade.dauphine.fr (Denis Bouyssou), marc.pirlot@fpms.ac.be (Marc Pirlot)
} 


\section{Introduction}

Building a preference relation on a set of alternatives evaluated on several attributes is the focal point of Multiple Criteria Decision Analysis (MCDA). The classical approach to achieve this goal consists in building a value function on the set of alternatives (Keeney and Raiffa 1976, Ch. 3). Since the construction of value functions is often demanding in terms of time and information, this approach cannot always be used in practice. For this reason, alternative methods have been proposed among which are the outranking methods. They compare alternatives in a pairwise manner and decide which is preferred on the basis of their evaluations on the several attributes. They mainly rest on "ordinal" considerations (for detailed presentations of these methods, we refer to Roy 1991, Roy and Bouyssou 1993).

Most outranking methods, including the well known ELECTRE methods, compare alternatives on the basis of the so-called concordance / non-discordance principle. It leads to accepting the proposition that an alternative is "superior" to another if:

- concordance condition: the coalition of attributes supporting it is "sufficiently important",

- non-discordance condition: there is no attribute that "strongly rejects" it.

In the ELECTRE methods, "superior" means "not worse". Such methods aim at building a reflexive preference relation that is interpreted as an "at least as good as" relation. In general, these relations may lack nice transitivity or completeness properties (on these issues, see Bouyssou 1992, 1996). The main goal of this paper is to characterize the reflexive binary relations that can be obtained on the basis of the concordance-discordance principle like in the ELECTRE I (Roy 1968) and ELECTRE II methods (Roy and Bertier 1973). In doing so, we build on previous results characterizing the relations that can be obtained only using the first part of the concordance / non-discordance principle, i.e., the concordance condition (Bouyssou and Pirlot 2005a, 2007b).

As first presented in Bouyssou, Pirlot, and Vincke (1997), the general strategy followed in this paper is to view outranking relations as a particular case of relations having a representation in the nontransitive decomposable models introduced in Bouyssou and Pirlot (1999, 2002b, 2004a); this was indeed our initial motivation for developing them. This particular case obtains when only a few distinct levels of preference differences are distinguished. This roughly leads to analyzing "ordinal aggregation" as an aggregation in which there are at most three types of preferences differences: positive, null and negative ones. This paper expands on this simple idea. It is organized as follows. Our setting is introduced in Section 2. Section 3 briefly recalls our previous results on concordance relations. We propose 
a characterization of reflexive concordance-discordance relations in Section 4. In Section 5, we specialize these results to impose some form of transitivity on each attribute. A final section discusses our results and positions them with respect to the existing literature on the subject.

\section{The setting}

\subsection{Binary relations}

We use a classical vocabulary for binary relations. A binary relation $\mathcal{R}$ on a set $A$ is a subset of $A \times A$. We mostly write $a \mathcal{R} b$ instead of $(a, b) \in \mathcal{R}$. The codual $\mathcal{R}^{c d}$ of $\mathcal{R}$ is the binary relation on $A$ such that, for all $a, b \in A$, $a \mathcal{R}^{c d} b \Leftrightarrow \operatorname{Not}[b \mathcal{R} a]$. Taking the codual of a relation is an involutive operation, i.e., the codual of the codual of a relation is this relation. It is easy to check that the codual of a complete relation is an asymmetric relation and conversely. The asymmetric part of a complete relation $\mathcal{R}$ is identical to its codual $\mathcal{R}^{c d}$.

A weak order (resp. an equivalence) is a complete and transitive (resp. reflexive, symmetric and transitive) binary relation. If $\mathcal{R}$ is an equivalence on $A, A / \mathcal{R}$ will denote the set of equivalence classes of $\mathcal{R}$ on $A$. When $\mathcal{R}$ is a weak order, it is clear that its symmetric part is an equivalence.

A semiorder is a reflexive, Ferrers (i.e., $[a \mathcal{R} b$ and $c \mathcal{R} d] \Rightarrow[a \mathcal{R} d$ or $c \mathcal{R} b]$ ) and semi-transitive (i.e., $[a \mathcal{R} b$ and $b \mathcal{R} c] \Rightarrow[a \mathcal{R} d$ or $d \mathcal{R} c]$ ) binary relation. An easy proof shows that a semiorder is complete, i.e., for all $a, b \in A, a \mathcal{R} b$ or $b \mathcal{R} a$.

A strict semiorder is an irreflexive, Ferrers, and semi-transitive binary relation. It is easy to check that a strict semiorder is an asymmetric binary relation. The codual of a semiorder is a strict semiorder and conversely.

As first observed by Luce (1956), any Ferrers and semitransitive relation $\mathcal{R}$ on $A$ induces a unique weak order $\mathcal{R}^{w o}$ on $A$ that is defined as follows:

$$
a \mathcal{R}^{w o} b \text { if } \forall c \in A,[b \mathcal{R} c \Rightarrow a \mathcal{R} c] \text { and }[c \mathcal{R} a \Rightarrow c \mathcal{R} b] .
$$

Suppose that $\mathcal{R}$ and $\mathcal{S}$ are two Ferrers and semitransitive relations. If $\mathcal{S}$ is the codual of $\mathcal{R}$, then it follows that $\mathcal{R}^{w o}=\mathcal{S}^{w o}$. Hence, the weak order induced by a semiorder is identical to the one induced by its asymmetric part.

Let $\mathcal{R}$ and $\mathcal{S}$ be two semiorders on $A$ such that $\mathcal{R} \subseteq \mathcal{S}$. We say that $(\mathcal{R}, \mathcal{S})$ is a nested chain of semiorders. Let $\mathcal{R}^{w o}$ (resp. $\mathcal{S}^{w o}$ ) be the weak order on $A$ induced by $\mathcal{R}$ (resp. $\mathcal{S}$ ). If the relation $\mathcal{R}^{w o} \cap \mathcal{S}^{w o}$ is complete (and therefore is a weak order), we say that $(\mathcal{R}, \mathcal{S})$ is a homogeneous nested chain of semiorders (Doignon, Monjardet, Roubens, and Vincke 1988).

We leave to the reader the easy proof of the following observation. 
Lemma 1. Let $\mathcal{R}$ be a binary relation on $A$. If there is a weak order $\mathcal{S}$ such that, for all $a, b, c \in A$,

$$
\begin{aligned}
& a \mathcal{R} b \text { and } b \mathcal{S} c \Rightarrow a \mathcal{R} c, \\
& a \mathcal{S} b \text { and } b \mathcal{R} c \Rightarrow a \mathcal{R} c
\end{aligned}
$$

then $\mathcal{R}$ is Ferrers and semitransitive. Furthermore, we have that $\mathcal{S} \subseteq \mathcal{R}^{\text {wo }}$.

\subsection{Notation and definitions}

In this paper $\succsim$ will always denote a reflexive binary relation on a set $X=$ $\prod_{i=1}^{n} X_{i}$ with $n \geq 2$. Elements of $X$ will be interpreted as alternatives evaluated on a set $N=\{1,2, \ldots, n\}$ of attributes and $\succsim$ as an "at least as good as" relation between these alternatives. We denote by $\succ($ resp. $\sim)$ the asymmetric (resp. symmetric) part of $\succsim$. A similar convention holds when $\succsim$ is starred, superscripted and/or subscripted.

For any nonempty subset $J$ of the set of attributes $N$, we denote by $X_{J}$ (resp. $\left.X_{-J}\right)$ the set $\prod_{i \in J} X_{i}$ (resp. $\left.\prod_{i \notin J} X_{i}\right)$. With customary abuse of notation, $\left(x_{J}, y_{-J}\right)$ will denote the element $w \in X$ such that $w_{i}=x_{i}$ if $i \in J$ and $w_{i}=y_{i}$ otherwise. We sometimes omit braces around sets. For instance, when $J=\{i\}$ we write $X_{-i}$ and $\left(x_{i}, y_{-i}\right)$.

We say that attribute $i \in N$ is influent (for $\succsim$ ) if there are $x_{i}, y_{i}, z_{i}, w_{i} \in X_{i}$ and $x_{-i}, y_{-i} \in X_{-i}$ such that $\left(x_{i}, x_{-i}\right) \succsim\left(y_{i}, y_{-i}\right)$ and $\left(z_{i}, x_{-i}\right) \succsim\left(w_{i}, y_{-i}\right)$ and degenerate otherwise. A degenerate attribute has no influence whatsoever on the comparison of the elements of $X$ and may be suppressed from $N$. As in Bouyssou and Pirlot (2005a), in order to avoid unnecessary minor complications, we suppose henceforth that all attributes in $N$ are influent.

\subsection{Concordance relations}

In Bouyssou and Pirlot (2005a), we have given a general definition of concordance relations and have shown that the preference relations produced by most of outranking methods fit into this framework, provided that no veto effect occurs (i.e., when only the concordance part of the concordance / non-discordance principle is used).

Definition 2 (Reflexive concordance relation). Let $\succsim$ be a reflexive binary relation on $X=\prod_{i=1}^{n} X_{i}$. We say that $\succsim$ is a reflexive concordance relation (or, more briefly, that $\succsim$ is an $R-C R$ ) if there are:

- a complete binary relation $S_{i}$ on each $X_{i}(i=1,2, \ldots, n)$,

- a binary relation $\unrhd$ between subsets of $N$ having $N$ for union that is monotonic w.r.t. inclusion, i.e., for all $A, B, C, D \subseteq N$ such that $A \cup B=N$ and $C \cup D=N$,

$$
[A \unrhd B, C \supseteq A, B \supseteq D] \Rightarrow C \unrhd D,
$$


such that, for all $x, y \in X$,

$$
x \succsim y \Leftrightarrow S(x, y) \unrhd S(y, x),
$$

where $S(x, y)=\left\{i \in N: x_{i} S_{i} y_{i}\right\}$. We say that $\left\langle\unrhd, S_{i}\right\rangle$ is a representation of $\succsim$ as an $R$-CR. Throughout the paper, $P_{i}$ (resp. $I_{i}$ ) will denote the asymmetric (resp. symmetric) part of $S_{i}$.

Let us illustrate this definition by a simple example.

Example 3 (Semiordered weighted majority). The binary relation $\succsim$ is a semiordered weighted majority preference relation if there are a real number $\varepsilon \geq 0$ and, for all $i \in N$, a semiorder $S_{i}$ on $X_{i}$, and a real number $w_{i}>0$, such that:

$$
x \succsim y \Leftrightarrow \sum_{i \in S(x, y)} w_{i} \geq \sum_{j \in S(y, x)} w_{j}-\varepsilon .
$$

Such a relation is easily seen to be a complete R-CR defining $\unrhd$ letting, for all $A, B \subseteq N$ such that $A \cup B=N$ :

$$
A \unrhd B \Leftrightarrow \sum_{i \in A} w_{i} \geq \sum_{j \in B} w_{j}-\varepsilon .
$$

When all relations $S_{i}$ are weak orders, $w_{i}=w_{j}$, for all $i, j \in N$, and $\varepsilon=0$, it is clear that the above model reduces to simple majority.

Observe that in the above example, all relations $S_{i}$ are supposed to be semiorders, whereas Definition 2 only requires them to be complete.

\subsection{Concordance-discordance relations}

ELECTRE I builds a reflexive concordance relation that is subsequently "censored" by imposing on it the non-discordance condition. This is illustrated below.

Example 4 (ELECTRE I, Roy 1968). In ELECTRE I, it is supposed that on each attribute $i \in N$, there are a real-valued function $u_{i}$ on $X_{i}$ and a pair of positive thresholds $p t_{i}$ and $v t_{i}$, with $p t_{i} \leq v t_{i}$. The relations $S_{i}$ and $V_{i}$ are defined letting, for all $x_{i}, y_{i} \in X_{i}$,

$$
\begin{aligned}
x_{i} S_{i} y_{i} & \Leftrightarrow u_{i}\left(x_{i}\right) \geq u_{i}\left(y_{i}\right)-p t_{i} \\
x_{i} V_{i} y_{i} & \Leftrightarrow u_{i}\left(x_{i}\right)>u_{i}\left(y_{i}\right)+v t_{i} .
\end{aligned}
$$

It is clear that $S_{i}$ is a semiorder. Similarly, the relation $V_{i}$ is a strict semiorder that is the asymmetric part of a semiorder $U_{i}$ such that, for all $x_{i}, y_{i} \in X_{i}$,

$$
x_{i} U_{i} y_{i} \Leftrightarrow \operatorname{Not}\left[y_{i} V_{i} x_{i}\right] \Leftrightarrow u_{i}\left(x_{i}\right) \geq u_{i}\left(y_{i}\right)-v t_{i}
$$


Since $v t_{i} \geq p t_{i}$, we have $S_{i} \subseteq U_{i}$, so that $P_{i} \supseteq V_{i}$. Hence, $\left(S_{i}, U_{i}\right)$ is a nested chain of semiorders. Using (1), consider the weak order $S_{i}{ }^{w o}$ associated to the semiorder $S_{i}$ and the weak order $U_{i}^{\text {wo }}$ associated to the semiorder $U_{i}$. From (4) and (5), it is clear that their intersection $T_{i}=U_{i}^{w o} \cap S_{i}{ }^{w o}$ is again a weak order. Hence, $\left(S_{i}, U_{i}\right)$ is a homogeneous nested chain of semiorders.

The binary relation $\succsim$ in ELECTRE I is built letting, for all $x, y \in X$,

$$
x \succsim y \Leftrightarrow \frac{\sum_{i \in S(x, y)} w_{i}}{\sum_{j \in N} w_{j}} \geq s \text { and } V(y, x)=\varnothing,
$$

where $V(y, x)=\left\{i \in N: y_{i} V_{i} x_{i}\right\}, w_{i}>0$, for all $i \in N$, and $s \in[1 / 2,1]$.

When all relations $V_{i}$ are empty, the relation $\succsim$ obtained with ELECTRE I is easily seen to be a R-CR with, for all $A, B \subseteq N$ such that $A \cup B=N$,

$$
A \unrhd B \Leftrightarrow \frac{\sum_{i \in A} w_{i}}{\sum_{j \in N} w_{j}} \geq s .
$$

When, this is not the case, the set $V(y, x)$ contains the attributes on which $y$ is "so much better" than $x$ that it is excluded to conclude that $x \succsim y$. Therefore, in ELECTRE I, $x$ is "at least as good as" $y$ if there is a majority of attributes on which $x$ is at least as good as $y$ (the attributes belonging to $S(x, y)$ should be "sufficiently important") and there is no attribute on which $x$ is "too much worse than" $y$ (the set $V(y, x)$ should be empty).

ELECTRE I is an example of what we call a reflexive concordance-discordance relation, a precise definition of which follows.

Definition 5 (Reflexive concordance-discordance relation). Let $\succsim$ be a reflexive binary relation on $X=\prod_{i=1}^{n} X_{i}$. We say that $\succsim$ is a reflexive concordancediscordance relation (or, more briefly, that $\succsim$ is an $R$-CDR) if there are:

- a complete binary relation $S_{i}$ on each $X_{i}(i=1,2, \ldots, n)$ (with asymmetric part $P_{i}$ and symmetric part $\left.I_{i}\right)$,

- an asymmetric binary relation $V_{i}$ on each $X_{i}(i=1,2, \ldots, n)$ such that $V_{i} \subseteq P_{i}$,

- a binary relation $\unrhd$ between subsets of $N$ having $N$ for union that is monotonic w.r.t. inclusion, i.e., such that (2) holds,

such that, for all $x, y \in X$,

$$
x \succsim y \Leftrightarrow[S(x, y) \unrhd S(y, x) \text { and } V(y, x)=\varnothing],
$$

where $S(x, y)=\left\{i \in N: x_{i} S_{i} y_{i}\right\}$ and $V(y, x)=\left\{i \in N: y_{i} V_{i} x_{i}\right\}$. We say that $\left\langle\unrhd, S_{i}, V_{i}\right\rangle$ is a representation of $\succsim$ as an $R-C D R$. 
Observe that the above definition only requires that, for all $i \in N, S_{i}$ is complete and $V_{i} \subseteq P_{i}$. In ELECTRE I much more is true since we know that $\left(S_{i}, U_{i}\right)$ is a homogeneous nested chain of semiorders. At this stage, this is not part of our definition.

\section{Background}

In this section, we briefly present the axiomatic framework and the previously obtained characterization of reflexive concordance relations within this framework.

\subsection{Conjoint measurement framework}

Concordance relations rely on comparing alternatives in pairwise manner on the basis of preference differences on each attribute. The relations defined below were introduced in Bouyssou and Pirlot (2002b). They will play a fundamental rôle in the sequel.

Definition 6 (Relations comparing preference differences). Let $\succsim$ be a binary relation on a set $X=\prod_{i=1}^{n} X_{i}$. We define the binary relations $\succsim_{i}^{*}$ and $\succsim_{i}^{* *}$ on $X_{i}^{2}$ letting, for all $x_{i}, y_{i}, z_{i}, w_{i} \in X_{i}$,

$$
\begin{aligned}
&\left(x_{i}, y_{i}\right) \succsim_{i}^{*}\left(z_{i}, w_{i}\right) \Leftrightarrow \\
& \quad\left[\text { for all } a_{-i}, b_{-i} \in X_{-i},\left(z_{i}, a_{-i}\right) \succsim\left(w_{i}, b_{-i}\right) \Rightarrow\left(x_{i}, a_{-i}\right) \succsim\left(y_{i}, b_{-i}\right)\right], \\
&\left(x_{i}, y_{i}\right) \succsim_{i}^{* *}\left(z_{i}, w_{i}\right) \Leftrightarrow\left[\left(x_{i}, y_{i}\right) \succsim_{i}^{*}\left(z_{i}, w_{i}\right) \text { and }\left(w_{i}, z_{i}\right) \succsim_{i}^{*}\left(y_{i}, x_{i}\right)\right] .
\end{aligned}
$$

The definition of $\succsim_{i}^{*}$ suggests that $\left(x_{i}, y_{i}\right) \succsim_{i}^{*}\left(z_{i}, w_{i}\right)$ can be interpreted as saying that the preference difference between $x_{i}$ and $y_{i}$ is at least as large as the preference difference between $z_{i}$ and $w_{i}$. The definition of $\succsim_{i}^{*}$ does not imply that the two "opposite" differences $\left(x_{i}, y_{i}\right)$ and $\left(y_{i}, x_{i}\right)$ are linked. This is at variance with the intuition concerning preference differences and motivates the introduction of the relation $\succsim_{i}^{* *}$. By construction, $\succsim_{i}^{*}$ and $\succsim_{i}^{* *}$ are always reflexive and transitive.

Definition 7 (Conditions $R C 1$ and $R C 2$ ). Let $\succsim$ be a binary relation on a set $X=\prod_{i=1}^{n} X_{i}$. This relation is said to satisfy:

$$
\begin{aligned}
& \left.R C 1_{i} \text { if } \begin{array}{c}
\left(x_{i}, a_{-i}\right) \succsim\left(y_{i}, b_{-i}\right) \\
\text { and } \\
\left(z_{i}, c_{-i}\right) \succsim\left(w_{i}, d_{-i}\right)
\end{array}\right\} \Rightarrow\left\{\begin{array}{c}
\left(x_{i}, c_{-i}\right) \succsim\left(y_{i}, d_{-i}\right) \\
\text { or } \\
\left(z_{i}, a_{-i}\right) \succsim\left(w_{i}, b_{-i}\right),
\end{array}\right. \\
& \left.R C 2_{i} \text { if } \begin{array}{c}
\left(x_{i}, a_{-i}\right) \succsim\left(y_{i}, b_{-i}\right) \\
\text { and } \\
\left(y_{i}, c_{-i}\right) \succsim\left(x_{i}, d_{-i}\right)
\end{array}\right\} \Rightarrow\left\{\begin{array}{c}
\left(z_{i}, a_{-i}\right) \succsim\left(w_{i}, b_{-i}\right) \\
\text { or } \\
\left(w_{i}, c_{-i}\right) \succsim\left(z_{i}, d_{-i}\right),
\end{array}\right.
\end{aligned}
$$

for all $x_{i}, y_{i}, z_{i}, w_{i} \in X_{i}$ and all $a_{-i}, b_{-i}, c_{-i}, d_{-i} \in X_{-i}$. We say that $\succsim$ satisfies $R C 1$ (resp. $R C 2$ ) if it satisfies $R C 1_{i}$ (resp. $R C 2_{i}$ ) for all $i \in N$. 
Condition $R C 1_{i}$ is equivalent to requiring that any two preference differences are comparable in terms of $\succsim_{i}^{*}$. Condition $R C 2_{i}$ imposes a "mirror effect" on the comparison of preference differences. This is summarized in the following:

Lemma 8 (Bouyssou and Pirlot 2002b, Lemma 1).

1. $R C 1_{i} \Leftrightarrow\left[\succsim_{i}^{*}\right.$ is complete $]$.

2. $R C 2_{i} \Leftrightarrow$ [for all $\left.x_{i}, y_{i}, z_{i}, w_{i} \in X_{i},\left(x_{i}, y_{i}\right) \beth_{i}^{*}\left(z_{i}, w_{i}\right) \Rightarrow\left(y_{i}, x_{i}\right) \succsim_{i}^{*}\left(w_{i}, z_{i}\right)\right]$.

3. $\left[R C 1_{i}\right.$ and $\left.R C 2_{i}\right] \Leftrightarrow\left[\succsim_{i}^{* *}\right.$ is complete $]$.

4. In the class of reflexive relations, $R C 1$ and $R C 2$ are independent conditions.

We consider binary relations $\succsim$ on $X$ that can be represented in the following model introduced in Bouyssou and Pirlot (2002b):

$$
x \succsim y \Leftrightarrow F\left(p_{1}\left(x_{1}, y_{1}\right), p_{2}\left(x_{2}, y_{2}\right), \ldots, p_{n}\left(x_{n}, y_{n}\right)\right) \geq 0,
$$

where $p_{i}$ are real-valued functions on $X_{i}^{2}$ that are skew symmetric (i.e., such that $p_{i}\left(x_{i}, y_{i}\right)=-p_{i}\left(y_{i}, x_{i}\right)$, for all $\left.x_{i}, y_{i} \in X_{i}\right)$ and $F$ is a real-valued function on $\prod_{i=1}^{n} p_{i}\left(X_{i}^{2}\right)$ being nondecreasing in all its arguments and such that, abusing notation, $F(\mathbf{0}) \geq 0$.

It is useful to interpret $p_{i}$ as a function measuring preference differences between levels on attribute $i \in N$. The fact that the functions $p_{i}$ are supposed to be skew symmetric means that the preference difference between $x_{i}$ and $y_{i}$ is the opposite of the preference difference between $y_{i}$ and $x_{i}$, which seems a reasonable hypothesis. In order to compare alternatives $x$ and $y$, model (M) proceeds as follows. On each attribute $i \in N$, the preference difference between $x_{i}$ and $y_{i}$ is measured using $p_{i}$. The synthesis of these preference differences is performed applying the function $F$ to the $p_{i}\left(x_{i}, y_{i}\right)$ 's. We then conclude that $x \succsim y$ when this synthesis is nonnegative. Given this interpretation, it seems reasonable to suppose that $F$ is nondecreasing in each of its arguments. The fact that $F(\mathbf{0}) \geq 0$ simply means that the synthesis of null preference differences on each attribute should be nonnegative; this ensures that $\succsim$ will be reflexive.

For finite or countably infinite sets, conditions $R C 1$ and $R C 2$ together with reflexivity are all that is needed in order to characterize model $(\mathrm{M})$. We have:

Theorem 9 (Bouyssou and Pirlot 2002b, Theorem 1). Let $\succsim$ be a binary relation on $X=\prod_{i=1}^{n} X_{i}$. If, for all $i \in N, X_{i}^{2} / \sim_{i}^{* *}$ is finite or countably infinite then $\succsim$ has a representation (M) if and only if (iff) it is reflexive and satisfies $R C 1$ and $R C 2$.

The extension of this result to the general case is easy but will not be useful here. 


\subsection{Concordance relations}

The general strategy used in Bouyssou and Pirlot (2005a, 2007b) to characterize concordance relations is to use model $(\mathrm{M})$ as a building block adding additional conditions ensuring that all functions $p_{i}$ take at most three distinct values. Hence, the "ordinal" character of the aggregation at work in concordance relations is modelled by saying that in an ordinal method, there can be at most three distinct types of preference differences: positive, null and negative ones. The additional conditions used in Bouyssou and Pirlot (2007b) to capture concordance relations are as follows.

Definition 10 (Conditions $M 1$ and $M 2$ ). Let $\succsim$ be a binary relation on a set $X=\prod_{i=1}^{n} X_{i}$. This relation is said to satisfy:

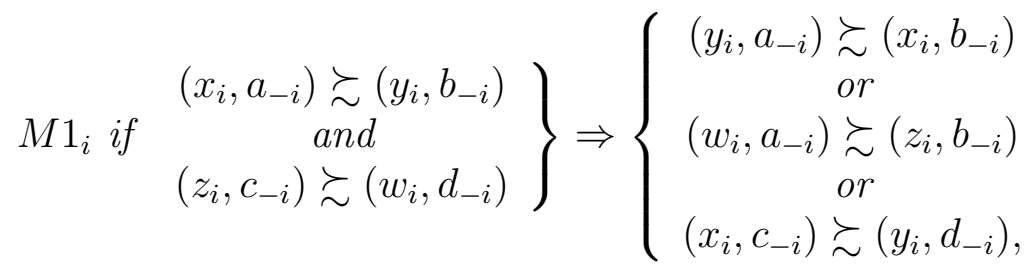

$$
\begin{aligned}
& \left.M 2_{i} \text { if } \begin{array}{rl}
\left(x_{i}, a_{-i}\right) & \succsim\left(y_{i}, b_{-i}\right) \\
\text { and } \\
\left(y_{i}, c_{-i}\right) \succsim\left(x_{i}, d_{-i}\right)
\end{array}\right\} \Rightarrow\left\{\begin{aligned}
\left(y_{i}, a_{-i}\right) & \succsim\left(x_{i}, b_{-i}\right) \\
& \text { or } \\
\left(z_{i}, a_{-i}\right) & \succsim\left(w_{i}, b_{-i}\right) \\
& \text { or } \\
\left(z_{i}, c_{-i}\right) & \succsim\left(w_{i}, d_{-i}\right),
\end{aligned}\right.
\end{aligned}
$$

for all $x_{i}, y_{i}, z_{i}, w_{i} \in X_{i}$ and all $a_{-i}, b_{-i}, c_{-i}, d_{-i} \in X_{-i}$. We say that $M 1$ (resp. M2) holds if $M 1_{i}$ (resp. $M 2_{i}$ ) holds for all $i \in N$.

It is not difficult to see that $M 1_{i}$ and $M 2_{i}$ drastically limit the possibility of distinguishing several classes of preference differences on each attribute using $\succsim_{i}^{*}$. Suppose for instance that the premises of $M 1_{i}$ holds and that its first conclusion is false. Because $\left(x_{i}, a_{-i}\right) \succsim\left(y_{i}, b_{-i}\right)$ and $\left(y_{i}, a_{-i}\right) \succsim\left(x_{i}, b_{-i}\right)$, it is clear that the preference difference $\left(y_{i}, x_{i}\right)$ is not larger (w.r.t. the relation $\left.\succsim_{i}^{*}\right)$ than its opposite preference difference $\left(x_{i}, y_{i}\right)$. In an R-CR, this can only happen if the difference $\left(x_{i}, y_{i}\right)$ is "positive" and, thus, the difference $\left(y_{i}, x_{i}\right)$ is "negative". But if the difference $\left(x_{i}, y_{i}\right)$ is "positive", there cannot exist a difference larger than $\left(x_{i}, y_{i}\right)$. Therefore if $\left(z_{i}, c_{-i}\right) \succsim\left(w_{i}, d_{-i}\right)$, we should obtain $\left(x_{i}, c_{-i}\right) \succsim\left(y_{i}, d_{-i}\right)$. This is what is required by $M 1_{i}$ (disregarding its second possible conclusion that only ensures that the condition will be independent from the ones used to characterize model (M)). Condition $M 2_{i}$ has a dual interpretation: if $\left(y_{i}, x_{i}\right)$ is not larger than its opposite preference difference $\left(x_{i}, y_{i}\right)$ then there can be no difference smaller than $\left(y_{i}, x_{i}\right)$. 
Remark 11. In Bouyssou and Pirlot (2005a), we used two conditions that are stronger than $M 1_{i}$ and $M 2_{i}$. It will prove useful to introduce them. The relation $\succsim$ is said to satisfy:

$$
\begin{aligned}
& \left.U C_{i} \text { if } \begin{array}{c}
\left(x_{i}, a_{-i}\right) \succsim\left(y_{i}, b_{-i}\right) \\
\text { and } \\
\left(z_{i}, c_{-i}\right) \succsim\left(w_{i}, d_{-i}\right)
\end{array}\right\} \Rightarrow\left\{\begin{array}{c}
\left(y_{i}, a_{-i}\right) \succsim\left(x_{i}, b_{-i}\right) \\
\text { or } \\
\left(x_{i}, c_{-i}\right) \succsim\left(y_{i}, d_{-i}\right),
\end{array}\right. \\
& \left.L C_{i} \text { if } \begin{array}{c}
\left(x_{i}, a_{-i}\right) \succsim\left(y_{i}, b_{-i}\right) \\
\text { and } \\
\left(y_{i}, c_{-i}\right) \succsim\left(x_{i}, d_{-i}\right)
\end{array}\right\} \Rightarrow\left\{\begin{array}{c}
\left(y_{i}, a_{-i}\right) \succsim\left(x_{i}, b_{-i}\right) \\
\text { or } \\
\left(z_{i}, c_{-i}\right) \succsim\left(w_{i}, d_{-i}\right),
\end{array}\right.
\end{aligned}
$$

for all $x_{i}, y_{i}, z_{i}, w_{i} \in X_{i}$ and all $a_{-i}, b_{-i}, c_{-i}, d_{-i} \in X_{-i}$.

Bouyssou and Pirlot (2005a, Lemma 16) and Bouyssou and Pirlot (2007b, Lemma 11) show that:

1. $U C_{i} \Rightarrow M 1_{i}$.

2. $L C_{i} \Rightarrow M 2_{i}$.

3. $U C_{i} \Leftrightarrow\left[\left(y_{i}, x_{i}\right) \beth_{i}^{*}\left(x_{i}, y_{i}\right) \Rightarrow\left(x_{i}, y_{i}\right) \succsim_{i}^{*}\left(z_{i}, w_{i}\right)\right.$, for all $\left.x_{i}, y_{i}, z_{i}, w_{i} \in X_{i}\right]$.

4. $L C_{i} \Leftrightarrow\left[\left(y_{i}, x_{i}\right) \beth_{i}^{*}\left(x_{i}, y_{i}\right) \Rightarrow\left(z_{i}, w_{i}\right) \succsim_{i}^{*}\left(y_{i}, x_{i}\right)\right.$, for all $\left.x_{i}, y_{i}, z_{i}, w_{i} \in X_{i}\right]$.

The problem with $U C$ and $L C$ is that they interact with $R C 1$ and $R C 2$ (see Bouyssou and Pirlot 2005a, Lemma 16). This explains our use of the slightly more involved conditions $M 1$ and $M 2$.

We have:

Theorem 12 (Bouyssou and Pirlot 2007b, Theorem 13). Let $\succsim$ be a binary relation on $X=\prod_{i=1}^{n} X_{i}$. Then $\succsim$ is an $R$-CR iff it is reflexive and satisfies $R C 1, R C 2$, $M 1$ and $M 2$. In the class of reflexive relations, conditions $R C 1, R C 2, M 1$ and M2 are independent.

This shows that the framework of model $(\mathrm{M})$ is adequate for analyzing R-CR. We show below that this is also the case for R-CDR.

\section{Reflexive concordance-discordance relations}

In an R-CDR (see Definition 5), the concordance condition is tempered by a non-discordance condition forbidding to have $x \succsim y$ when there is one attribute on which $y$ is far better than $x$. In terms of preference differences, this adds the possibility of having two categories of negative differences: normal ones (acting as in an R-CR) and intolerable ones (corresponding to a veto).

We first show that an R-CDR is always a particular case of model (M) (conditions $R C 1, R C 2$ hold) and that, as in an R-CDR, there can be only one type of positive differences (condition $M 1$ holds). 
Lemma 13. If $\succsim$ is an $R$-CDR then it satisfies $R C 1, R C 2$ and $M 1$.

Proof. $[R C 1]$ Let $\left\langle\unrhd, S_{i}, V_{i}\right\rangle$ be a representation of $\succsim$. Suppose that $\left(x_{i}, a_{-i}\right) \succsim$ $\left(y_{i}, b_{-i}\right),\left(z_{i}, c_{-i}\right) \succsim\left(w_{i}, d_{-i}\right)$. This implies that $\operatorname{Not}\left[\begin{array}{lll}y_{i} & V_{i} & x_{i}\end{array}\right]$ and $\operatorname{Not}\left[\begin{array}{lll}w_{i} & V_{i} & z_{i}\end{array}\right]$. Suppose that $y_{i} P_{i} x_{i}$. The definition of an R-CDR implies that $\left(z_{i}, a_{-i}\right) \succsim$ $\left(w_{i}, b_{-i}\right)$. If $x_{i} P_{i} y_{i}$, the definition of an R-CDR implies that $\left(x_{i}, c_{-i}\right) \succsim\left(y_{i}, d_{-i}\right)$. Suppose now that $x_{i} I_{i} y_{i}$. If $z_{i} S_{i} w_{i}$, we obtain, using the definition of an RCDR, $\left(z_{i}, a_{-i}\right) \succsim\left(w_{i}, b_{-i}\right)$. If $w_{i} P_{i} z_{i}$, using the definition of an R-CDR leads to $\left(x_{i}, c_{-i}\right) \succsim\left(y_{i}, d_{-i}\right)$.

[RC2] Suppose that $\left(x_{i}, a_{-i}\right) \succsim\left(y_{i}, b_{-i}\right),\left(y_{i}, c_{-i}\right) \succsim\left(x_{i}, d_{-i}\right)$. This implies that $\operatorname{Not}\left[\begin{array}{lll}y_{i} & V_{i} & x_{i}\end{array}\right]$ and $\operatorname{Not}\left[\begin{array}{lll}x_{i} & V_{i} & y_{i}\end{array}\right]$. Suppose that $x_{i} S_{i} y_{i}$. If $z_{i} S_{i} w_{i}$, we know that $\operatorname{Not}\left[w_{i} V_{i} z_{i}\right]$ and the definition of an R-CDR leads to $\left(z_{i}, a_{-i}\right) \succsim\left(w_{i}, b_{-i}\right)$. If $w_{i} S_{i} z_{i}$, we know that $\operatorname{Not}\left[z_{i} V_{i} w_{i}\right]$. The definition of an R-CDR leads to $\left(w_{i}, c_{-i}\right) \succsim\left(z_{i}, d_{-i}\right)$. The proof is similar if we suppose that $y_{i} S_{i} x_{i}$.

[M1] Suppose that $\left(x_{i}, a_{-i}\right) \succsim\left(y_{i}, b_{-i}\right)$ and $\left(z_{i}, c_{-i}\right) \succsim\left(w_{i}, d_{-i}\right)$. This implies that $\operatorname{Not}\left[y_{i} V_{i} x_{i}\right]$ and $\operatorname{Not}\left[w_{i} V_{i} z_{i}\right]$. If $y_{i} S_{i} x_{i}$, we know that $\operatorname{Not}\left[x_{i} V_{i} y_{i}\right]$ so that we have $\left(y_{i}, a_{-i}\right) \succsim\left(x_{i}, b_{-i}\right)$, using the definition of an R-CDR. If $x_{i} P_{i} y_{i}$, we know that $\operatorname{Not}\left[\begin{array}{lll}y_{i} & V_{i} & x_{i}\end{array}\right]$ so that we have $\left(x_{i}, c_{-i}\right) \succsim\left(y_{i}, d_{-i}\right)$, using the definition of an R-CDR.

In view of Lemma 13 and Theorem 12, it is clear that that condition $M 2$ may be violated in an R-CDR. This is due to the possible presence of a veto effect: it may happen that the premise of $M 2_{i}$ is fulfilled while the conclusion is false because the pair $\left(w_{i}, z_{i}\right)$ belongs to $V_{i}$. This motivates the introduction of the following condition that weakens $M 2_{i}$. The work of Greco, Matarazzo, and Słowiński (2001a) has been inspiring in devising it.

Definition 14 (Condition M3). Let $\succsim$ be a binary relation on a set $X=\prod_{i=1}^{n} X_{i}$. This relation is said to satisfy:

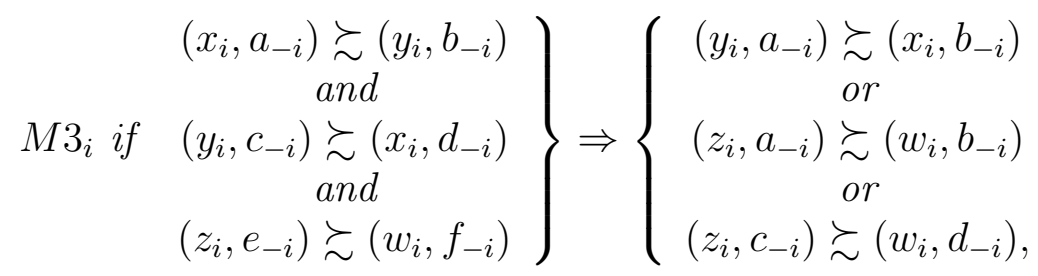

for all $x_{i}, y_{i}, z_{i}, w_{i} \in X_{i}$ and all $a_{-i}, b_{-i}, c_{-i}, d_{-i}, e_{-i}, f_{-i} \in X_{-i}$. We say that $\succsim$ satisfies $M 3$ if it satisfies $M 3_{i}$ for all $i \in N$.

The meaning of $M 3_{i}$ can be intuitively expressed as follows. Suppose that the three premises of $M 3_{i}$ hold and that its first conclusion is false (we disregard the second possible conclusion of $M 3_{i}$, the rôle of which is to ensure that the condition is independent from the ones needed to characterize model $(\mathrm{M})$ ). 
This implies that the difference $\left(y_{i}, x_{i}\right)$ is "negative" since it is not larger than its opposite difference $\left(x_{i}, y_{i}\right)$. In an R-CDR a negative preference difference is the smallest among all preference differences that do not correspond to a veto. Because $\left(z_{i}, e_{-i}\right) \succsim\left(w_{i}, f_{-i}\right)$, we know that $\operatorname{Not}\left[w_{i} V_{i} z_{i}\right]$. Hence, the difference $\left(z_{i}, w_{i}\right)$ is not smaller than the difference $\left(y_{i}, x_{i}\right)$, so that $\left(y_{i}, c_{-i}\right) \succsim\left(x_{i}, d_{-i}\right)$ implies $\left(z_{i}, c_{-i}\right) \succsim\left(w_{i}, d_{-i}\right)$. More formally, we have:

Lemma 15. If $\succsim$ is an $R$-CDR then it satisfies $M 3$.

Proof. Suppose that $\left(x_{i}, a_{-i}\right) \succsim\left(y_{i}, b_{-i}\right),\left(y_{i}, c_{-i}\right) \succsim\left(x_{i}, d_{-i}\right)$ and $\left(z_{i}, e_{-i}\right) \succsim\left(w_{i}, f_{-i}\right)$. By construction, we know that $\operatorname{Not}\left[x_{i} V_{i} y_{i}\right], \operatorname{Not}\left[\begin{array}{lll}y_{i} & V_{i} & x_{i}\end{array}\right]$ and $\operatorname{Not}\left[\begin{array}{lll}w_{i} & V_{i} & z_{i}\end{array}\right]$. If $y_{i} S_{i} x_{i}$, the definition of an R-CDR implies $\left(y_{i}, a_{-i}\right) \succsim\left(x_{i}, b_{-i}\right)$. If $x_{i} P_{i} y_{i}$, the definition of an R-CDR implies $\left(z_{i}, c_{-i}\right) \succsim\left(w_{i}, d_{-i}\right)$. Hence $M 3_{i}$ is fulfilled.

Remark 16. It is clear that $M 2_{i}$ implies $M 3_{i}$, since $M 3_{i}$ is obtained from $M 2_{i}$ by adding the premise $\left(z_{i}, e_{-i}\right) \succsim\left(w_{i}, f_{-i}\right)$. Bouyssou and Pirlot (2007a, Example 39) show that there are reflexive relations satisfying $R C 1, R C 2, M 1$ on all attributes and $M 3_{i}$ on all but one attribute. In view of Theorem 12 , this shows that conditions $R C 1, R C 2, M 1$ and $M 3$ are independent in the class of reflexive relations.

The following lemma analyzes the structure of the relation $\succsim_{i}^{*}$ under $R C 1$, $R C 2, M 1$ and $M 3$. It shows that when there are two distinct types of negative differences, the smallest ones can be interpreted as a veto.

Lemma 17. Let $\succsim$ be a binary relation on $X=\prod_{i=1}^{n} X_{i}$. If $\succsim$ satisfies $R C 1$, $R C 2, M 1$ and $M 3$, then, for all $x_{i}, y_{i}, z_{i}, w_{i}, r_{i}, s_{i} \in X_{i}$,

1. $\left(x_{i}, y_{i}\right) \succ_{i}^{*}\left(y_{i}, x_{i}\right) \Rightarrow\left(x_{i}, y_{i}\right) \succsim_{i}^{*}\left(z_{i}, w_{i}\right)$.

2. $\left[\left(x_{i}, y_{i}\right) \succ_{i}^{*}\left(y_{i}, x_{i}\right) \succ_{i}^{*}\left(z_{i}, w_{i}\right)\right] \Rightarrow\left(r_{i}, s_{i}\right) \succsim_{i}^{*}\left(z_{i}, w_{i}\right)$. Furthermore, we have $\left(z_{i}, a_{-i}\right) \nsucceq\left(w_{i}, b_{-i}\right)$, for all $a_{-i}, b_{-i} \in X_{-i}$.

Proof. Part 1 follows from results obtained in Bouyssou and Pirlot (2007b): by Lemma 11.3 in this paper, we know that $R C 2_{i}$ and $M 1_{i}$ imply $U C_{i}$, i.e., that $\left(y_{i}, x_{i}\right) \Varangle_{i}^{*}\left(x_{i}, y_{i}\right) \Rightarrow\left(x_{i}, y_{i}\right) \succsim_{i}^{*}\left(z_{i}, w_{i}\right)$, for all $x_{i}, y_{i}, z_{i}, w_{i} \in X_{i}$ (see Remark 11). Since $R C 1_{i}$ is equivalent to the fact that $\succsim_{i}^{*}$ is complete, $\left(y_{i}, x_{i}\right) \beth_{i}^{*}\left(x_{i}, y_{i}\right)$ implies $\left(x_{i}, y_{i}\right) \succ_{i}^{*}\left(y_{i}, x_{i}\right)$, which proves Part 1 .

Part 2. Suppose that, for some $x_{i}, y_{i}, z_{i}, w_{i}, r_{i}, s_{i} \in X_{i}$, we have $\left(x_{i}, y_{i}\right) \succ_{i}^{*}$ $\left(y_{i}, x_{i}\right) \succ_{i}^{*}\left(z_{i}, w_{i}\right)$ and $\left(z_{i}, w_{i}\right) \succ_{i}^{*}\left(r_{i}, s_{i}\right)$. This implies $\left(x_{i}, a_{-i}\right) \succsim\left(y_{i}, b_{-i}\right)$, $\left(y_{i}, a_{-i}\right) \nsucceq\left(x_{i}, b_{-i}\right),\left(y_{i}, c_{-i}\right) \succsim\left(x_{i}, d_{-i}\right),\left(z_{i}, c_{-i}\right) \nsucceq\left(w_{i}, d_{-i}\right)$ and $\left(z_{i}, e_{-i}\right) \succsim$ $\left(w_{i}, f_{-i}\right),\left(r_{i}, e_{-i}\right) \succsim\left(s_{i}, f_{-i}\right)$, for some $a_{-i}, b_{-i}, c_{-i}, d_{-i}, e_{-i}, f_{-i} \in X_{-i}$. Using $M 3_{i}$, $\left(x_{i}, a_{-i}\right) \succsim\left(y_{i}, b_{-i}\right),\left(y_{i}, c_{-i}\right) \succsim\left(x_{i}, d_{-i}\right),\left(z_{i}, e_{-i}\right) \succsim\left(w_{i}, f_{-i}\right),\left(y_{i}, a_{-i}\right) \succsim\left(x_{i}, b_{-i}\right)$ and $\left(z_{i}, c_{-i}\right) \succsim\left(w_{i}, d_{-i}\right)$ imply that $\left(z_{i}, a_{-i}\right) \succsim\left(w_{i}, b_{-i}\right)$. This leads to $\left(y_{i}, c_{-i}\right) \succsim$ $\left(x_{i}, d_{-i}\right),\left(z_{i}, c_{-i}\right) \succsim\left(w_{i}, d_{-i}\right),\left(z_{i}, a_{-i}\right) \succsim\left(w_{i}, b_{-i}\right)$ and $\left(y_{i}, a_{-i}\right) \succsim\left(x_{i}, b_{-i}\right)$, contradicting the completeness of $\succsim_{i}^{*}$ that follows from $R C 1_{i}$. Note that the contradiction 
is obtained as soon as $\left(z_{i}, e_{-i}\right) \succsim\left(w_{i}, f_{-i}\right)$, for some $e_{-i}, f_{-i} \in X_{-i}$. This proves the second part of the assertion.

For relations satisfying $R C 1, R C 2, M 1$ and $M 3$, we define a relation $S_{i}$ on $X_{i}$, using $\succsim_{i}^{*}$ for comparing each difference $\left(x_{i}, y_{i}\right)$ to its "opposite" difference $\left(y_{i}, x_{i}\right)$. Similarly, we introduce the veto relation $V_{i}$ when a difference is strictly smaller (in terms of $\succ_{i}^{*}$ ) than two opposite differences. We have:

Lemma 18. Let $\succsim$ be a binary relation on $X=\prod_{i=1}^{n} X_{i}$ satisfying $R C 1, R C 2$, $M 1$ and $M 3$.

1. The relation $S_{i}$ on $X_{i}$ defined letting $x_{i} S_{i} y_{i}$ iff $\left(x_{i}, y_{i}\right) \succsim_{i}^{*}\left(y_{i}, x_{i}\right)$ is complete.

2. If $x_{i} I_{i} y_{i}$ and $z_{i} I_{i} w_{i}$ then $\left(x_{i}, y_{i}\right) \sim_{i}^{*}\left(z_{i}, w_{i}\right) \sim_{i}^{*}\left(y_{i}, x_{i}\right) \sim_{i}^{*}\left(w_{i}, z_{i}\right) \sim_{i}^{*}$ $\left(a_{i}, a_{i}\right)$, for all $a_{i} \in X_{i}$.

3. If $z_{i} P_{i} w_{i}$ and $x_{i} P_{i} y_{i}$ then $\left(z_{i}, w_{i}\right) \sim_{i}^{*}\left(x_{i}, y_{i}\right)$. Furthermore, $P_{i}$ is nonempty.

4. Define the relation $V_{i}$ on $X_{i}$ letting $x_{i} V_{i} y_{i}$ if, for some $z_{i}, w_{i} \in X_{i},\left(z_{i}, w_{i}\right) \succ_{i}^{*}$ $\left(w_{i}, z_{i}\right) \succ_{i}^{*}\left(y_{i}, x_{i}\right)$. We have:

(a) $V_{i} \subseteq P_{i}$,

(b) $\left[x_{i} V_{i} y_{i}\right.$ and $\left.z_{i} V_{i} w_{i}\right] \Rightarrow\left(y_{i}, x_{i}\right) \sim_{i}^{*}\left(w_{i}, z_{i}\right)$,

(c) $\left[x_{i} P_{i} y_{i}, z_{i} P_{i} w_{i}, \operatorname{Not}\left[x_{i} V_{i} y_{i}\right]\right.$ and $\left.\operatorname{Not}\left[z_{i} V_{i} w_{i}\right]\right] \Rightarrow\left(y_{i}, x_{i}\right) \sim_{i}^{*}\left(w_{i}, z_{i}\right)$.

Proof. Part 1. We have $x_{i} S_{i} y_{i}$ iff $\left(x_{i}, y_{i}\right) \succsim_{i}^{*}\left(y_{i}, x_{i}\right)$. Since $\succsim_{i}^{*}$ is complete due to $R C 1$, it follows that $S_{i}$ is complete.

Part 2. Using the definition of $S_{i}, x_{i} I_{i} y_{i}$ and $z_{i} I_{i} w_{i}$ is equivalent to $\left(x_{i}, y_{i}\right) \sim_{i}^{*}$ $\left(y_{i}, x_{i}\right)$ and $\left(z_{i}, w_{i}\right) \sim_{i}^{*}\left(w_{i}, z_{i}\right)$. The conclusion follows from $R C 2$.

Part 3. Suppose that $z_{i} P_{i} w_{i}$ and $x_{i} P_{i} y_{i}$, so that $\left(x_{i}, y_{i}\right) \succ_{i}^{*}\left(y_{i}, x_{i}\right)$ and $\left(z_{i}, w_{i}\right) \succ_{i}^{*}\left(w_{i}, z_{i}\right)$. Lemma 17.1 implies that $\left(z_{i}, w_{i}\right) \succsim_{i}^{*}\left(x_{i}, y_{i}\right)$ and $\left(x_{i}, y_{i}\right) \succsim_{i}^{*}$ $\left(z_{i}, w_{i}\right)$ so that $\left(z_{i}, w_{i}\right) \sim_{i}^{*}\left(x_{i}, y_{i}\right)$.

If $P_{i}$ is empty, we must have for all $x_{i}, y_{i}, z_{i}, w_{i} \in X_{i}, x_{i} I_{i} y_{i}$ and $z_{i} I_{i} w_{i}$. Using Lemma 18.2, this implies $\left(x_{i}, y_{i}\right) \sim_{i}^{*}\left(z_{i}, w_{i}\right)$, violating the fact that attribute $i \in N$ is influent.

Part 4a. We have $x_{i} V_{i} y_{i}$ iff $\left(z_{i}, w_{i}\right) \succ_{i}^{*}\left(w_{i}, z_{i}\right) \succ_{i}^{*}\left(y_{i}, x_{i}\right)$. Suppose that $\operatorname{Not}\left[x_{i} P_{i} y_{i}\right]$ so that $\left(y_{i}, x_{i}\right) \succsim_{i}^{*}\left(x_{i}, y_{i}\right)$. Using $R C 1$ and $R C 2$, it is easy to check that $\left(z_{i}, w_{i}\right) \succ_{i}^{*}\left(w_{i}, z_{i}\right)$ implies $\left(z_{i}, w_{i}\right) \succsim_{i}^{*}\left(a_{i}, a_{i}\right) \succsim_{i}^{*}\left(w_{i}, z_{i}\right)$, for all $a_{i} \in X_{i}$. We therefore obtain $\left(a_{i}, a_{i}\right) \succ_{i}^{*}\left(y_{i}, x_{i}\right) \succsim_{i}^{*}\left(x_{i}, y_{i}\right)$. This contradicts $R C 2$.

Part 4b. Suppose that $x_{i} V_{i} y_{i}$ and $z_{i} V_{i} w_{i}$. Lemma 17.2 implies that $\left(w_{i}, z_{i}\right) \succsim_{i}^{*}$ $\left(y_{i}, x_{i}\right)$ and $\left(y_{i}, x_{i}\right) \succsim_{i}^{*}\left(w_{i}, z_{i}\right)$ so that $\left(w_{i}, z_{i}\right) \sim_{i}^{*}\left(y_{i}, x_{i}\right)$.

Part 4c. By definition, we have $\left(x_{i}, y_{i}\right) \succ_{i}^{*}\left(y_{i}, x_{i}\right)$ and $\left(z_{i}, w_{i}\right) \succ_{i}^{*}\left(w_{i}, z_{i}\right)$. Suppose that $\left(y_{i}, x_{i}\right) \succ_{i}^{*}\left(w_{i}, z_{i}\right)$. This would imply $\left(x_{i}, y_{i}\right) \succ_{i}^{*}\left(y_{i}, x_{i}\right) \succ_{i}^{*}\left(w_{i}, z_{i}\right)$, contradicting the fact that $\operatorname{Not}\left[z_{i} V_{i} w_{i}\right]$. Similarly it is impossible that $\left(w_{i}, z_{i}\right) \succ_{i}^{*}$ $\left(y_{i}, x_{i}\right)$. Hence, we have $\left(y_{i}, x_{i}\right) \sim_{i}^{*}\left(w_{i}, z_{i}\right)$.

This leads to our characterization of R-CDR. 
Theorem 19. Let $\succsim$ be a reflexive binary relation on $X=\prod_{i=1}^{n} X_{i}$. Then $\succsim$ is an $R$-CDR iff it satisfies $R C 1, R C 2, M 1$ and $M 3$. These axioms are independent in the class of reflexive binary relations.

Proof. Necessity results from Lemmas 13 and 15. The independence of the axioms results from Remark 16. We show sufficiency.

Define the relation $S_{i}$ on $X_{i}$ letting $x_{i} S_{i} y_{i}$ if $\left(x_{i}, y_{i}\right) \succsim_{i}^{*}\left(y_{i}, x_{i}\right)$. Lemma 18.1 implies that $S_{i}$ is complete. Lemma 18.3 implies that $P_{i}$ is not empty.

Define the relation $V_{i}$ on $X_{i}$ letting $x_{i} V_{i} y_{i}$ if, for some $z_{i}, w_{i} \in X_{i},\left(z_{i}, w_{i}\right) \succ_{i}^{*}$ $\left(w_{i}, z_{i}\right) \succ_{i}^{*}\left(y_{i}, x_{i}\right)$. Lemma 18.4 implies that $V_{i} \subseteq P_{i}$.

Consider two subsets $A, B \subseteq N$ such that $A \cup B=N$ and let:

$$
\begin{gathered}
A \unrhd B \Leftrightarrow \\
{[x \succsim y, \text { for some } x, y \in X \text { such that } S(x, y)=A \text { and } S(y, x)=B] .}
\end{gathered}
$$

Suppose that $x \succsim y$. Lemma 17.2 implies that $V(y, x)=\varnothing$. By construction, we have $S(x, y) \unrhd S(y, x)$.

Suppose now that $V(y, x)=\varnothing$ and $S(x, y) \unrhd S(y, x)$. Let us show that we have $x \succsim y$. By construction, $S(x, y) \unrhd S(y, x)$ implies that there are $z, w \in X$ such that $z \succsim w, S(x, y)=S(z, w)$ and $S(y, x)=S(w, z)$. For all $i \in N$ such that $z_{i} I_{i} w_{i}$, we have $x_{i} I_{i} y_{i}$ and Lemma 18.4 implies $\left(x_{i}, y_{i}\right) \sim_{i}^{*}\left(z_{i}, w_{i}\right)$. For all $i \in N$ such that $z_{i} P_{i} w_{i}$, we have $x_{i} P_{i} y_{i}$ and Lemma 18.1 implies $\left(x_{i}, y_{i}\right) \sim_{i}^{*}\left(z_{i}, w_{i}\right)$. For all $i \in N$ such that $w_{i} P_{i} z_{i}$, we have $y_{i} P_{i} x_{i}$. By hypothesis, we have $\operatorname{Not}\left[y_{i} V_{i} x_{i}\right]$. Because $z \succsim w$, we have $\operatorname{Not}\left[w_{i} V_{i} z_{i}\right]$. Lemma 18.4c implies that $\left(x_{i}, y_{i}\right) \sim_{i}^{*}\left(z_{i}, w_{i}\right)$. Hence, we have $\left(x_{i}, y_{i}\right) \sim_{i}^{*}\left(z_{i}, w_{i}\right)$, for all $i \in N$, so that $z \succsim w$ implies $x \succsim y$.

It remains to show that $\unrhd$ is monotonic. Suppose that $A \unrhd B$, so that, for some $x, y \in X, S(x, y)=A, S(y, x)=B$ and $x \succsim y$. Since $x \succsim y$, we know that $N o t\left[y_{i} V_{i} x_{i}\right]$, for all $i \in N$. Let $A^{\prime}=A \backslash B, B^{\prime}=B \backslash A$ and $C=A \cap B$. Suppose that $Z \supseteq A, B \supseteq W$ and $Z \cup W=N$. Let $Z^{\prime}=Z \backslash W, W^{\prime}=W \backslash Z$ and $Y=Z \cap W$. It is clear that $i \in A^{\prime}$ implies $i \in Z^{\prime}$. If $i \in C$, we have either $i \in Z^{\prime}$ or $i \in Y$. We divide $C$ into $D$ and $E$ according to whether we have $i \in Z^{\prime}$ or $i \in Y$. Let $i \in B^{\prime}$, we have either $i \in Z^{\prime}, i \in Y$ or $i \in W^{\prime}$. We divide $B^{\prime}$ into $F, G$ and $H$ according to whether we have $i \in Z^{\prime}, i \in Y$ or $i \in W^{\prime}$. Therefore, we have $Z^{\prime}=A^{\prime} \cup D \cup F, Y=E \cup G$ and $W^{\prime}=H$.

Because, each attribute is influent, we know that for all $i \in N$, we can find $a_{i}, b_{i} \in X_{i}$ such that $a_{i} P_{i} b_{i}$. Let us build the alternatives $\alpha, \beta \in X$ according to the following table:

$$
\begin{array}{ccccccc} 
& A^{\prime} & D & E & F & G & H \\
\alpha & x_{i} & a_{i} & x_{i} & a_{i} & a_{i} & x_{i} \\
\beta & y_{i} & b_{i} & y_{i} & b_{i} & a_{i} & y_{i}
\end{array}
$$


It is easy to check that we have $\left(\alpha_{i}, \beta_{i}\right) \succsim_{i}^{*}\left(x_{i}, y_{i}\right)$, for all $i \in N$, so that $x \succsim y$ implies $\alpha \succsim \beta$. By construction, we have $S(\alpha, \beta)=C$ and $S(\beta, \alpha)=D$, so that $C \unrhd D$.

Bouyssou and Pirlot (2005a, Lemma 2) show that, when all attributes are influent, the representation $\left\langle\unrhd, S_{i}\right\rangle$ of an R-CR is always unique. Such a result does not hold for the representation $\left\langle\underline{\unrhd}, S_{i}, V_{i}\right\rangle$ of an R-CDR. Indeed suppose that on some attribute $i \in N$, it is impossible that $x \succsim y$ as soon as $y_{i} P_{i} x_{i}$, i.e., $\left(y_{i}, x_{i}\right) \succ_{i}^{*}\left(x_{i}, y_{i}\right)$. This can be represented saying that it is never true that $A \unrhd B$ when $i \in B$ together with $V_{i}=\varnothing$. Alternatively, we may take the relation $V_{i}$ to hold as soon as $\left(y_{i}, x_{i}\right) \succ_{i}^{*}\left(x_{i}, y_{i}\right)$.

The first option has been taken in the proof of Theorem 19. This leads to building a representation $\left\langle\unrhd, S_{i}, V_{i}\right\rangle$ of an R-CDR that uses a minimal amount of veto. We do not investigate here the additional conditions under which the representation $\left\langle\unrhd, S_{i}, V_{i}\right\rangle$ would be unique since this does not seem to add insight on the nature of these relations. These conditions have to do with the fact that for all attributes there are levels linked by $S_{i}$ but not by $V_{i}$. Note however that assessment methods designed for R-CDR should be prepared to deal with this lack of uniqueness.

\section{Concordance-discordance relations with attribute transitivity}

Our definition of R-CDR in Section 4 does not require the relations $S_{i}$ or $V_{i}$ to possess any remarkable property besides the completeness of $S_{i}$ and the fact that $V_{i} \subseteq P_{i}$. This is at variance with what is done in most outranking methods (see the examples in Section 2.4). In this section, we show how to characterize R-CDR with the following additional requirements:

- the relation $S_{i}$ is a semiorder,

- the veto relation $V_{i}$ is a strict semiorder such that $V_{i} \subseteq P_{i}$,

- $\left(S_{i}, U_{i}\right)$ is a homogeneous nested chain of semiorders, where $U_{i}$ denotes the semiorder having $V_{i}$ for asymmetric part,

for all $i \in N$. As discussed in Section 2.4, this will bring us quite close to the models that are used in practice.

As a first step, we show how to refine the framework provided by model (M).

\subsection{Conjoint measurement framework continued}

We first show, following Bouyssou and Pirlot (2004a), how to introduce a linear arrangement of the elements of each $X_{i}$ within the framework of model (M). The following relations play a fundamental rôle. 
Definition 20 (Relations comparing levels on each attribute). Let $\succsim$ be a binary relation on a set $X=\prod_{i=1}^{n} X_{i}$. We define the binary relations $\succsim_{i}^{+}, \succsim_{i}^{-}$and $\succsim_{i}^{ \pm}$ on $X_{i}$ letting, for all $x_{i}, y_{i} \in X_{i}$,

$$
\begin{aligned}
& x_{i} \succsim_{i}^{+} y_{i} \Leftrightarrow \forall a_{-i} \in X_{-i}, b \in X,\left[\left(y_{i}, a_{-i}\right) \succsim b \Rightarrow\left(x_{i}, a_{-i}\right) \succsim b\right], \\
& x_{i} \succsim_{i}^{-} y_{i} \Leftrightarrow \forall a \in X, b_{-i} \in X_{-i},\left[a \succsim\left(x_{i}, b_{-i}\right) \Rightarrow a \succsim\left(y_{i}, b_{-i}\right)\right], \\
& x_{i} \succsim_{i}^{ \pm} y_{i} \Leftrightarrow x_{i} \succsim_{i}^{+} y_{i} \text { and } x_{i} \succsim_{i}^{-} y_{i} .
\end{aligned}
$$

This leads to:

Lemma 21 (Bouyssou and Pirlot 2005b, Lemma 3.8). For all $i \in N$ and all $x_{i}, y_{i} \in X_{i}$, we have:

$$
\begin{gathered}
x_{i} \succsim_{i}^{+} y_{i} \Leftrightarrow\left[\forall z_{i} \in X_{i},\left(x_{i}, z_{i}\right) \succsim_{i}^{*}\left(y_{i}, z_{i}\right)\right], \\
x_{i} \succsim_{i}^{-} y_{i} \Leftrightarrow\left[\forall w_{i} \in X_{i},\left(w_{i}, y_{i}\right) \succsim_{i}^{*}\left(w_{i}, x_{i}\right)\right], \\
x_{i} \succsim_{i}^{ \pm} y_{i} \Leftrightarrow \\
\forall z_{i} \in X_{i},\left(x_{i}, z_{i}\right) \succsim_{i}^{*}\left(y_{i}, z_{i}\right) \text { and } \forall w_{i} \in X_{i},\left(w_{i}, y_{i}\right) \succsim_{i}^{*}\left(w_{i}, x_{i}\right) .
\end{gathered}
$$

By construction, the relations $\succsim_{i}^{+}, \succsim_{i}^{-}$and $\succsim_{i}^{ \pm}$are always reflexive and transitive. Their completeness is related to the following axioms.

Definition 22 (Conditions $A C 1, A C 2$ and $A C 3$ ). We say that $\succsim$ satisfies:

$$
\begin{gathered}
\left.A C 1_{i} \text { if } \begin{array}{c}
x \succsim y \\
\text { and } \\
z \succsim w
\end{array}\right\} \Rightarrow\left\{\begin{array}{c}
\left(z_{i}, x_{-i}\right) \succsim y \\
\text { or } \\
\left(x_{i}, z_{-i}\right) \succsim w,
\end{array}\right. \\
\left.A C 2_{i} \text { if } \begin{array}{c}
x \succsim y \\
\text { and } \\
z \succsim w
\end{array}\right\} \Rightarrow\left\{\begin{array}{c}
x \succsim\left(w_{i}, y_{-i}\right) \\
\text { or } \\
z \succsim\left(y_{i}, w_{-i}\right),
\end{array}\right. \\
\left.\begin{array}{c}
z \succsim\left(c_{i}, a_{-i}\right) \\
\text { and } \\
\left(c_{i}, b_{-i}\right) \succsim y
\end{array}\right\} \Rightarrow\left\{\begin{array}{c}
z \succsim\left(d_{i}, a_{-i}\right) \\
\text { or } \\
\left(d_{i}, b_{-i}\right) \succsim y,
\end{array}\right.
\end{gathered}
$$

for all $x, y, z, w \in X$, all $a_{-i}, b_{-i} \in X_{-i}$ and all $c_{i}, d_{i} \in X_{i}$. We say that $\succsim$ satisfies $A C 1$ (resp. AC2, AC3) if it satisfies $A C 1_{i}$ (resp. $A C 2_{i}, A C 3_{i}$ ) for all $i \in N$.

These three conditions are transparent variations on the theme of the Ferrers $(A C 1$ and $A C 2$ ) and semi-transitivity $(A C 3)$ conditions that are made possible by the product structure of $X$. They are directly related to properties of relations $\succsim_{i}^{+}, \succsim_{i}^{-}$and $\succsim_{i}^{ \pm}$as stated in the next lemma.

\section{Lemma 23.}


1. $A C 1_{i} \Leftrightarrow \succsim_{i}^{+}$is complete.

2. $A C 2_{i} \Leftrightarrow \succsim_{i}^{-}$is complete.

3. $A C 3_{i} \Leftrightarrow\left[\operatorname{Not}\left[x_{i} \succsim_{i}^{+} y_{i}\right] \Rightarrow y_{i} \succsim_{i}^{-} x_{i}\right] \Leftrightarrow\left[\operatorname{Not}\left[x_{i} \succsim_{i}^{-} y_{i}\right] \Rightarrow y_{i} \succsim_{i}^{+} x_{i}\right]$.

4. $\left[A C 1_{i}, A C 2_{i}\right.$ and $\left.A C 3_{i}\right] \Leftrightarrow \succsim_{i}^{ \pm}$is complete.

5. In the class of reflexive relations satisfying $R C 1$ and $R C 2, A C 1, A C 2$ and $A C 3$ are independent conditions.

Proof. See Bouyssou and Pirlot (2004b, Lemma 3) for Parts 1 to 4 and Bouyssou and Pirlot (2004a, Section 5.1.2) for Part 5.

In Bouyssou and Pirlot (2004a), we consider binary relations $\succsim$ on $X$ that can be represented as:

$$
x \succsim y \Leftrightarrow F\left(\varphi_{1}\left(u_{1}\left(x_{1}\right), u_{1}\left(y_{1}\right)\right), \ldots, \varphi_{n}\left(u_{n}\left(x_{n}\right), u_{n}\left(y_{n}\right)\right)\right) \geq 0,
$$

where $u_{i}$ are real-valued functions on $X_{i}, \varphi_{i}$ are real-valued functions on $u_{i}\left(X_{i}\right)^{2}$ that are skew symmetric, nondecreasing in their first argument (and, therefore, nonincreasing in their second argument) and $F$ is a real-valued function on $\prod_{i=1}^{n} \varphi_{i}\left(u_{i}\left(X_{i}\right)^{2}\right)$ being nondecreasing in all its arguments and such that $F(\mathbf{0}) \geq 0$.

Going from model (M) to model ( $\left.\mathrm{M}^{*}\right)$ amounts to requiring that each function $p_{i}$ measuring preference differences can be factorized as $\varphi_{i}\left(u_{i}\left(x_{i}\right), u_{i}\left(y_{i}\right)\right)$ thereby reflecting an underlying linear arrangement of the elements of $X_{i}$.

The conditions introduced so far allow to characterize model $\left(\mathrm{M}^{*}\right)$ when each $X_{i}$ is at most countably infinite. We have:

Theorem 24 (Bouyssou and Pirlot 2004a, Theorem 2). Let $\succsim$ be a binary relation on a finite or countably infinite set $X=\prod_{i=1}^{n} X_{i}$. Then $\succsim$ has a representation $\left(\mathrm{M}^{*}\right)$ if and only if it is reflexive and satisfies $R C 1, R C 2, A C 1, A C 2$ and $A C 3$.

Remark 25. Note that, contrary to Theorem 19, Theorem 24 is only stated here for finite or countably infinite sets $X$. This is no mistake: we refer to Bouyssou and Pirlot (2004a) for details and for the analysis of the extension of this result to the general case.

Although model $\left(\mathrm{M}^{*}\right)$ is a particular case of model $(\mathrm{M})$, it is still flexible enough to contain as particular cases models like the additive value function model (Krantz, Luce, Suppes, and Tversky 1971) or Tversky's additive difference model (Tversky 1969). We show below that it also contains all R-CDR in which the relations $S_{i}$ and $U_{i}$ are semiorders and $\left(S_{i}, U_{i}\right)$ form a homogeneous nested chain of semiorders. 


\section{2. $R-C D R$ with attribute transitivity}

Let us first precisely define what we will call $R$-CDR with attribute transitivity (R-CDR-AT).

Definition 26 (R-CDR with attribute transitivity). An $R$-CDR with attribute transitivity $(R-C D R-A T)$ is an $R-C D R$ for which, for all $i \in N$ :

- $S_{i}$ is a semiorder with asymmetric part $P_{i}$,

- $V_{i}$ is the asymmetric part of a semiorder $U_{i}$ with $U_{i} \supseteq S_{i}$ and, hence, $V_{i} \subseteq P_{i}$,

- $\left(S_{i}, U_{i}\right)$ form a homogeneous chain of semiorders, i.e., there is a weak order $R_{i}$ on $X_{i}$ such that:

$$
\begin{aligned}
& \qquad x_{i} R_{i} y_{i} \Rightarrow \forall z_{i} \in X_{i},\left[y_{i} S_{i} z_{i} \Rightarrow x_{i} S_{i} z_{i}\right] \text { and }\left[z_{i} S_{i} x_{i} \Rightarrow z_{i} S_{i} y_{i}\right] \text {, } \\
& \text { and } \\
& \qquad x_{i} R_{i} y_{i} \Rightarrow \forall z_{i} \in X_{i},\left[y_{i} U_{i} z_{i} \Rightarrow x_{i} U_{i} z_{i}\right] \text { and }\left[z_{i} U_{i} x_{i} \Rightarrow z_{i} U_{i} y_{i}\right] .
\end{aligned}
$$

It is easy to check that when $R_{i}$ satisfies (11) and (12), the same relations hold with $P_{i}$ instead of $S_{i}$ and $V_{i}$ instead of $U_{i}$. The following lemma shows that all $\mathrm{R}$-CDR-AT have a representation in model $\left(\mathrm{M}^{*}\right)$.

Lemma 27. If $\succsim$ is an $R$-CDR-AT then $\succsim$ satisfies $A C 1, A C 2$ and $A C 3$.

Proof. Let $\left\langle\unrhd, S_{i}, V_{i}\right\rangle$ be a representation of the R-CDR-AT $\succsim$ and let $R_{i}$ be the weak order obtained by intersecting the weak orders induced by the semiorders $S_{i}$ and $U_{i}$.

$\left[A C 1_{i}\right]$. Suppose that $\left(x_{i}, x_{-i}\right) \succsim\left(y_{i}, y_{-i}\right)$ and $\left(z_{i}, z_{-i}\right) \succsim\left(w_{i}, w_{-i}\right)$. We want to show that either $\left(z_{i}, x_{-i}\right) \succsim\left(y_{i}, y_{-i}\right)$ or $\left(x_{i}, z_{-i}\right) \succsim\left(w_{i}, w_{-i}\right)$.

From the hypothesis, we get that $\operatorname{Not}\left[\begin{array}{lll}y_{i} & V_{i} & x_{i}\end{array}\right]$ and $\operatorname{Not}\left[\begin{array}{lll}w_{i} & V_{i} & z_{i}\end{array}\right]$. Since $R_{i}$ is a weak order, either $x_{i} R_{i} z_{i}$ or $z_{i} R_{i} x_{i}$. Suppose $z_{i} R_{i} x_{i}$ and observe that this together with $\operatorname{Not}\left[\begin{array}{lll}y_{i} & V_{i} & x_{i}\end{array}\right]$ implies $\operatorname{Not}\left[\begin{array}{lll}y_{i} & V_{i} & z_{i}\end{array}\right]$. We show that $\left(x_{i}, x_{-i}\right) \succsim$ $\left(y_{i}, y_{-i}\right)$ implies $\left(z_{i}, x_{-i}\right) \succsim\left(y_{i}, y_{-i}\right)$. If $y_{i} P_{i} x_{i}$, the conclusion follows from the monotonicity of $\unrhd$. If $x_{i} S_{i} y_{i}$, we also have $z_{i} S_{i} y_{i}$ (using $z_{i} R_{i} x_{i}$ and (11)), hence the conclusion follows from the monotonicity of $\unrhd$ (if $x_{i} I_{i} y_{i}$ ) or directly (if $\left.x_{i} P_{i} y_{i}\right)$. The case in which $x_{i} R_{i} z_{i}$ leads to proving that $\left(x_{i}, z_{-i}\right) \succsim\left(w_{i}, w_{-i}\right)$ in an analogous manner. Hence $A C 1_{i}$ holds. The proof for $A C 2_{i}$ is similar.

$\left[A C 3_{i}\right]$. Suppose that $\left(z_{i}, z_{-i}\right) \succsim\left(x_{i}, a_{-i}\right)$ and $\left(x_{i}, b_{-i}\right) \succsim\left(y_{i}, y_{-i}\right)$. We want to show that either $\left(z_{i}, z_{-i}\right) \succsim\left(w_{i}, a_{-i}\right)$ or $\left(w_{i}, b_{-i}\right) \succsim\left(y_{i}, y_{-i}\right)$.

The hypothesis implies $\operatorname{Not}\left[x_{i} V_{i} z_{i}\right]$ and $\operatorname{Not}\left[y_{i} V_{i} x_{i}\right]$. We have either $x_{i} R_{i} w_{i}$ or $w_{i} R_{i} x_{i}$. Assume the former. Together with $\operatorname{Not}\left[x_{i} V_{i} z_{i}\right]$ this entails $\operatorname{Not}\left[w_{i} V_{i}\right.$ $z_{i}$. As for $A C 1_{i}$, one easily shows that $\left(z_{i}, z_{-i}\right) \succsim\left(x_{i}, a_{-i}\right)$ and $x_{i} R_{i} w_{i}$ yield $\left(z_{i}, z_{-i}\right) \succsim\left(w_{i}, a_{-i}\right)$. The proof, assuming $w_{i} R_{i} x_{i}$, is similar. 
We have:

Lemma 28. If $\succsim$ is an $R$-CDR that satisfies $A C 1, A C 2$ and $A C 3$ then it is an $R-C D R-A T$.

Proof. Define the relations $S_{i}$ and $V_{i}$ as in Lemma 18. Define the relation $U_{i}$ as the codual of $V_{i}$. The proof will be complete if we show that $\left(S_{i}, U_{i}\right)$ is a nested homogeneous chain of semiorders. Because $A C 1, A C 2$ and $A C 3$ hold, we know that $\succsim_{i}^{ \pm}$is a weak order. We claim that the proof will be complete if we show that, for all $x_{i}, y_{i}, z_{i} \in X_{i}$ :

$$
\begin{aligned}
& x_{i} \succsim_{i}^{ \pm} y_{i} \text { and } y_{i} S_{i} z_{i} \Rightarrow x_{i} S_{i} z_{i}, \\
& x_{i} S_{i} y_{i} \text { and } y_{i} \succsim_{i}^{ \pm} z_{i} \Rightarrow x_{i} S_{i} z_{i}, \\
& x_{i} \succsim_{i}^{ \pm} y_{i} \text { and } y_{i} V_{i} z_{i} \Rightarrow x_{i} S_{i} z_{i}, \\
& x_{i} V_{i} y_{i} \text { and } y_{i} \succsim_{i}^{ \pm} z_{i} \Rightarrow x_{i} S_{i} z_{i},
\end{aligned}
$$

Indeed, using Lemma 1, this will imply that both $S_{i}$ and $V_{i}$ are Ferrers and semitransitive. Using Lemma 18.1, we know that $S_{i}$ is reflexive. Using Lemma 18.4, we know that $V_{i}$ is asymmetric. Hence, $S_{i}$ will be a semiorder and $V_{i}$ a strict semiorder. Lemma 1 implies that $\succsim_{i}^{ \pm}$is included in both the weak order associated to $S_{i}$ and the weak order associated to $V_{i}$. We know that the weak order associated to $V_{i}$ is identical to the weak order associated to $U_{i}$. Hence, we know that $\succsim_{i}^{ \pm} \subseteq S_{i}^{w o} \cap U_{i}^{w o}$. Lemma 18.4a implies that $V_{i} \subseteq P_{i}$, so that $S_{i} \subseteq U_{i}$. Hence, $\left(S_{i}, U_{i}\right)$ will be a nested homogeneous chain of semiorders.

Let us show that (13) holds. Suppose that $x_{i} \succsim_{i}^{ \pm} y_{i}$ and $y_{i} S_{i} z_{i}$. Since $x_{i} \succsim_{i}^{ \pm} y_{i}$, we know from $(10)$ that $\left(x_{i}, w_{i}\right) \succsim_{i}^{*}\left(y_{i}, w_{i}\right)$ and $\left(w_{i}, y_{i}\right) \succsim_{i}^{*}\left(w_{i}, x_{i}\right)$, for all $w_{i} \in X_{i}$. By definition, we know that $y_{i} S_{i} z_{i}$ implies $\left(y_{i}, z_{i}\right) \succsim_{i}^{*}\left(z_{i}, y_{i}\right)$. Hence, we have $\left(x_{i}, z_{i}\right) \succsim_{i}^{*}\left(y_{i}, z_{i}\right),\left(y_{i}, z_{i}\right) \succsim_{i}^{*}\left(z_{i}, y_{i}\right)$, and $\left(z_{i}, y_{i}\right) \succsim_{i}^{*}\left(z_{i}, x_{i}\right)$, so that $\left(x_{i}, z_{i}\right) \succsim_{i}^{*}\left(z_{i}, x_{i}\right)$, using the transitivity of $\succsim_{i}^{*}$. This implies $x_{i} S_{i} z_{i}$. The proof for (14) is similar.

Let us show that (15) holds. Suppose that $x_{i} \succsim_{i}^{ \pm} y_{i}$ and $y_{i} V_{i} z_{i}$. Since $x_{i} \succsim_{i}^{ \pm} y_{i}$, we know from $(10)$ that $\left(x_{i}, w_{i}\right) \succsim_{i}^{*}\left(y_{i}, w_{i}\right)$ and $\left(w_{i}, y_{i}\right) \succsim_{i}^{*}\left(w_{i}, x_{i}\right)$, for all $w_{i} \in X_{i}$. By definition, we know that $y_{i} V_{i} z_{i}$ implies $\left(a_{i}, b_{i}\right) \succ_{i}^{*}\left(b_{i}, a_{i}\right) \succ_{i}^{*}\left(z_{i}, y_{i}\right)$, for some $a_{i}, b_{i} \in X_{i}$. Since $\left(z_{i}, y_{i}\right) \succsim_{i}^{*}\left(z_{i}, x_{i}\right)$, we obtain that $\left(a_{i}, b_{i}\right) \succ_{i}^{*}\left(b_{i}, a_{i}\right) \succ_{i}^{*}\left(z_{i}, x_{i}\right)$, so that $x_{i} V_{i} z_{i}$. The proof for (16) is similar.

This leads to our characterization of R-CDR-AT.

Theorem 29. Let $\succsim$ be a binary relation on $X=\prod_{i=1}^{n} X_{i}$. Then $\succsim$ is an $R-C D R$ $A T$ iff it is reflexive and satisfies $R C 1, R C 2, M 1, M 3, A C 1, A C 2$ and $A C 3$. In the class of reflexive relations, these conditions are independent. 
Proof. The characterization of R-CDR-AT results immediately from Lemma 27, Theorem 19 and Lemma 28. In view of proving the independence of the axioms, we need seven examples. They are given in Bouyssou and Pirlot (2007a).

In Bouyssou and Pirlot (2005a, 2007b), we have studied reflexive concordance relations with attribute transitivity (R-CR-AT). These relations are just R-CR admitting a representation $\left\langle\unrhd, S_{i}\right\rangle$ in which all $S_{i}$ are semiorders. These are clearly a special case of R-CDR-AT (Definition 26) in which all $V_{i}$ are empty relations. The characterization of R-CR-AT only differs from that of R-CDR-AT by the substitution of axiom $M 3$ by axiom $M 2$ and the omission of $A C 2$, which, in presence of the other conditions is equivalent to $A C 1$ (Bouyssou and Pirlot 2005a, Lemma 27).

\section{Discussion}

We have given several axiomatic characterizations of outranking relations based on the concordance / non-discordance principle. This was done in a traditional conjoint measurement setting ${ }^{1}$, i.e., using a binary relation defined on a Cartesian product as the only primitive. This leads to conditions entirely phrased in terms of $\succsim$. These conditions could well be subjected to empirical tests (we have not yet started investigating this point). Furthermore, the proofs of our results are constructive, which may be useful to devise assessment protocols. In a companion paper (Bouyssou and Pirlot 2007a), we have investigated the relations between outranking methods, such as TACTIC, leading to an asymmetric relation, interpreted as strict preference, and R-CDR. This leads to several new insights and, in particular to the study of outranking methods in which there is no veto effect but a dual "bonus" effect. Finally, it should be mentioned that our tools and results seem to be well suited to guide the development of outranking methods in which the non-discordance condition would be interpreted in less radical a way than in traditional methods exclusively relying on the idea of veto. For instance, one could well consider methods in which the conjunction of several negative differences would play the rôle of a veto, whereas none of them would do so on their own (see Bouyssou et al. 1997).

We would like to conclude with the mention of some limitations of the present study and its relation to the literature. We refer to Bouyssou and Pirlot (2005a)

\footnotetext{
${ }^{1}$ Different primitives are used in Pirlot (1997) who uses concepts from social choice and, hence, views outranking methods as techniques aggregating information available on each attribute. For a detailed comparison of the conjoint measurement approach followed here and the approach based on social choice concepts, we refer to Bouyssou, Marchant, Pirlot, Tsoukiàs, and Vincke (2006, Ch. 4-6).
} 
for other elements of discussion, including the important issue of the sweeping consequences of imposing nice transitivity properties to outranking relations obtained using the concordance / non-discordance principle.

\subsection{Limitations and directions for future work}

The practitioner of outranking methods will surely have noticed a number of limitations of the present work. We mention here what we consider to be the most important ones.

Our emphasis has been on outranking methods that lead to the construction of "crisp" preference relations. This is a severe limitation since many well known outranking methods, like ELECTRE III (Roy 1978) or PROMETHEE (Brans and Mareschal 2002) lead to the construction of valued relations, i.e., relations in which a number is attached to each ordered pair of alternatives reflecting the credibility or intensity of the underlying preference statement. Strictly speaking, this paper does not bring anything to the study of such methods. However, let us mention that it is possible to extend models $(\mathrm{M})$ and $\left(\mathrm{M}^{*}\right)$ to cover the case of valued binary relations: instead of comparing the value of $F$ to a fixed threshold (0), this value can be seen as defining the valued relation. This calls for further study.

With the ELECTRE methods in mind, a limitation of our work is that it does not take "weak preference" (interpreted as an hesitation between indifference and strict preference) into account. This is also a severe limitation since "weak preference" plays an important part in some outranking methods. Nevertheless, as pointed out in Tsoukiàs, Perny, and Vincke (2002), modelling "hesitation" is not an easy task and may necessitate the use of non-classical logics. Hence, it would be accurate to say that our results only deal with "idealized" outranking methods in which "hesitation" plays no rôle. Nevertheless our feeling is that these idealized methods remain close in spirit to the real outranking methods and that our conditions capture some of the central features of the latter.

It may also seem that our model for concordance remains too general. Indeed, in most methods weights are attached to each attribute and their sum are used to perform the concordance test. Our models do not use weights at all and are simply based on an importance relation between coalitions of attributes. We do not consider this limitation as very serious. On the practical side, dispensing with additive weights is indeed feasible, e.g., making use of symbolic inference techniques inspired from the Artificial Intelligence field: this was convincingly demonstrated with the "rough set" approach to MCDA, as presented, e.g., in Greco, Matarazzo, and Słowiński (2001b, 2005). On the theoretical side, formulating conditions ensuring that the relation $\unrhd$ has an additive representation is not a difficult task. Completing it is unlikely to lead to conditions giving much insight on the underlying methods: since $N$ is finite, these conditions will require a denumerable scheme of conditions that cannot be truncated. 
A final important limitation of our work is that it is limited to the so-called "construction phase" of outranking methods. Since the relations obtained as the result of this construction phase do not, in general, possess remarkable properties of transitivity or completeness, using them to devise a recommendation is not an easy task and calls for the application of specific techniques (this is the so-called "exploitation phase", see Roy and Bouyssou 1993, Chap. 6). We have little to say on how an axiomatic analysis that would include both the construction and the exploitation phases of outranking methods could be conducted. This is all the more true that there is no general agreement on what are the "best" techniques to derive a recommendation on the basis of an outranking relation in a given problem formulation. The sorting problem formulation seems the most easy one to deal with: since it only uses the comparison of alternatives with carefully selected profiles, intransitivity and incompleteness are not central issues. Results of this kind have been obtained in Bouyssou and Marchant (2007a,b). The analysis of both the construction and exploitation phases of outranking methods clearly calls for more research (first results were obtained in Bouyssou et al. 2006, Ch. 5, in a social choice framework).

\subsection{Relation to the literature}

This paper is not the first attempt to analyze the concordance / non-discordance principle in a conjoint measurement perspective. In what follows, we try to position our contribution w.r.t. this earlier literature.

\subsubsection{The approach using noncompensation}

The work of Bouyssou and Vansnick (1986) on TACTIC is probably the first attempt to tackle the problem studied here. This paper uses a classical conjoint measurement setting to analyze asymmetric concordance relations like the one built in TACTIC (Vansnick 1986). The central condition used in this paper to characterize such relations is a condition called "noncompensation" that was introduced in Fishburn $(1975,1976)$. It says that, for all $x, y, z, w \in X$,

$$
\left.\begin{array}{l}
\succ(x, y)=\succ(z, w) \\
\succ(y, x)=\succ(w, z)
\end{array}\right\} \Rightarrow[x \succ y \Leftrightarrow z \succ w]
$$

where $\succ(x, y)=\left\{i \in N: x_{i} \succ_{i} y_{i}\right\}$ and $\succ_{i}$ is the marginal binary relation on $X_{i}$ induced by $\succ$, i.e., the relation such that, for all $x_{i}, y_{i} \in X_{i}$,

$$
x_{i} \succ_{i} y_{i} \Leftrightarrow\left[\left(x_{i}, a_{-i}\right) \succ\left(y_{i}, a_{-i}\right), \text { for all } a_{-i} \in X_{-i}\right] .
$$

When coupled with a suitable monotonicity condition, or when strengthened as:

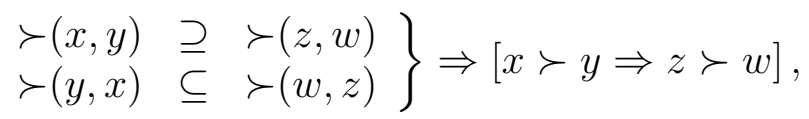


the noncompensation condition seems to offer an interesting basis to analyze the concordance principle. However, as discussed in Bouyssou and Pirlot (2005a, Sect. 5.2) this approach to concordance does not allow to deal with the whole variety of concordance relations. Furthermore, this approach is not well suited to characterize a relation $\succ$ in which the relations $\succ_{i}$ would have nice transitivity properties. Finally, the noncompensation condition is very specific to "ordinal" methods. Using it does not allow to characterize concordance relation within a broader framework that also encompasses other types of relations.

Bouyssou and Vansnick (1986) have approached the introduction of discordance via the following weakening of the noncompensation condition saying that, for all $x, y, z, w \in X$,

$$
\left.\begin{array}{l}
\succ(x, y)=\succ(z, w) \\
\succ(y, x)=\succ(w, z)
\end{array}\right\} \Rightarrow[x \succ y \Rightarrow w \nsucc z] .
$$

When $\succ(x, y)=\succ(z, w)$ and $\succ(y, x)=\succ(w, z)$ and $x \succ y$, this new condition allows to have either $z \succ w$ or $z \sim w$, where $\sim$ is seen here as the symmetric complement of $\succ$. It is not difficult to see that TACTIC satisfies this new condition. Indeed, the original noncompensation condition is (up to the point made earlier) satisfied for the concordance part of the method. The effect of discordance is to transform $\succ$ relations into $\sim$ ones. Hence, TACTIC satisfies condition (19).

There are several problems with this approach (that motivated one of the author of the present paper to abandon it and to develop the material presented here). We already mentioned that it is not flexible enough to cover all concordance relations of interest. We also noted that it is not well suited to introduce a linear arrangement of elements on each $X_{i}$. This last problem becomes even more important when discordance comes into play and there is a need to introduce links between $\succ_{i}$ and $V_{i}$. The route followed in Bouyssou and Vansnick (1986) to tackle this problem is correct but somewhat ad hoc.

A final and major limitation of this approach is that, contrary to the approach taken here, it does not generalize outside the realm of asymmetric relations (in Bouyssou and Pirlot (2007a), we discuss at length the duality relations between asymmetric and reflexive concordance-relations). As first noted in Bouyssou (1986, 1992) it is simple to reformulate the noncompensation condition so that it becomes adapted to the treatment of "at least as good as" relations. This leads to a condition of the type:

$$
\left.\begin{array}{l}
\succsim(x, y)=\succsim(z, w) \\
\succsim(y, x)=\succsim(w, z)
\end{array}\right\} \Rightarrow[x \succsim y \Leftrightarrow z \succsim w],
$$

where $\succsim(x, y)=\left\{i \in N: x_{i} \succsim_{i} y_{i}\right\}$ and $\succsim_{i}$ is the marginal binary relation on $X_{i}$ induced by $\succsim$, i.e., the relation such that, for all $x_{i}, y_{i} \in X_{i}$,

$$
x_{i} \succsim_{i} y_{i} \Leftrightarrow\left[\left(x_{i}, a_{-i}\right) \succsim\left(y_{i}, a_{-i}\right) \text {, for all } a_{-i} \in X_{-i}\right] .
$$


This approach was later developed in Dubois, Fargier, Perny, and Prade (2003). As was the case with the original noncompensation condition, (20) does not allow to deal with the whole variety of concordance relations (see Bouyssou and Pirlot 2005a, Examples 30 and 31), is very specific to this type of relations, and is not very well suited to introduce a linear arrangement of the elements on each $X_{i}$. Even worse, the route followed by Bouyssou and Vansnick (1986) in order to cope with veto effects does not work here. Mimicking the above approach, we could consider a condition saying that

$$
\left.\begin{array}{l}
\succsim(x, y)=\succsim(z, w) \\
\succsim(y, x)=\succsim(w, z)
\end{array}\right\} \Rightarrow[x \succ y \Rightarrow w \nsucc z]
$$

but even such a weak condition fails with most outranking relations. Indeed, an indifference situation between two alternatives obtained on the sole basis of the concordance test may be broken in an unpredictable way by veto effects. This leads to possible violations of condition (21). It is easy to build examples, e.g., using ELECTRE I, in which one has $\succsim(x, y)=\succsim(z, w), \succsim(y, x)=\succsim(w, z), x \sim y$ and $z \sim w$ when only the concordance part of the method is used. Introducing veto effects, one can easily obtain $x \succ y$ and $w \succ z$, violating (21).

The approach using variants of noncompensation is often seen to have some advantages w.r.t. the approach used here. First it has been shown (see Bouyssou 1992, or Dubois et al. 2003) to be particularly well suited to transfer "Arrow-like" results (i.e., results showing that requiring $\succsim$ to have "nice properties" induces a very undesirable repartition of "importance" among the various attributes) to the context of MCDA. We have shown in Bouyssou and Pirlot (2002a) that a similar analysis can also be performed in our more general framework, which leads to even more powerful results (this also shows that the hope of devising outranking methods based on the idea of concordance that would always lead to binary relations possessing nice transitivity properties is unfortunately somewhat chimeric). Noncompensation-like conditions are also often seen as being "simpler" and "more natural" than the type of conditions used here. We disagree. Besides the fact that "simplicity" is a very subjective criterion, we would like to point out that we have strived to present conditions that are entirely phrased using our primitives, i.e., the relation $\succsim$ on $X$. If one tries to reformulate the noncompensation condition with such a constraint, the result does not appear to be much simpler than our conditions.

\subsubsection{The approach of Greco et al. (2001a)}

Greco et al. (2001a) give results that are closely related to the ones presented here. Motivated by the ELECTRE I method, their aim was to characterize the particular class of R-CDR in which the relation $\unrhd$ is such that, for all $A, B \subseteq N$ 
such that $A \cup B=N$,

$$
A \unrhd B \Rightarrow A \unrhd N
$$

It is easy to see that this additional condition is satisfied in ELECTRE I (see Example 4). As we did above, the approach of Greco et al. (2001a) is based on conditions limiting the number of distinct equivalence classes of $\succsim_{i}^{*}$ (see Bouyssou et al. 1997).

When discordance is not taken into account, the central condition used by Greco et al. (2001a) is the following. We say that $\succsim$ is super-coarse on attribute $i \in N$ if, for all $x_{i}, y_{i}, z_{i}, w_{i}, r_{i}, s_{i} \in X_{i}$ and all $a_{-i}, b_{-i}, c_{-i}, d_{-i} \in X_{-i}$,

$$
\left.\begin{array}{c}
\left(x_{i}, a_{-i}\right) \underset{\text { and }}{\text { and }}\left(y_{i}, b_{-i}\right) \\
\left(z_{i}, c_{-i}\right) \succsim\left(w_{i}, d_{-i}\right)
\end{array}\right\} \Rightarrow\left\{\begin{array}{c}
\left(x_{i}, c_{-i}\right) \succsim\left(y_{i}, d_{-i}\right) \\
\text { or } \\
\left(r_{i}, a_{-i}\right) \succsim\left(s_{i}, b_{-i}\right) .
\end{array}\right.
$$

This condition is clear strengthening of $R C 1$. It is not difficult to see that a relation $\succsim$ is super-coarse on attribute $i \in N$ if and only if $\succsim_{i}^{*}$ is complete and has at most two distinct equivalence classes.

On its own, super-coarseness does not imply independence (in our framework independence is ensured via the use of $R C 2)$. Therefore nothing prevents $\left(x_{i}, x_{i}\right)$ and $\left(y_{i}, y_{i}\right)$ from belonging to two distinct equivalence classes of $\succsim_{i}^{*}$. In order to characterize R-CR satisfying (22), Greco et al. (2001a) use super-coarseness as well as an additional condition saying that, for all $i \in N$, all $x_{i}, y_{i}, w_{i} \in X_{i}$ and all $a_{-i}, b_{-i} \in X_{-i}$,

$$
\left(x_{i}, a_{-i}\right) \succsim\left(y_{i}, b_{-i}\right) \Rightarrow\left(w_{i}, a_{-i}\right) \succsim\left(w_{i}, b_{-i}\right) .
$$

This is a rather strong condition implying at the same time independence and the fact that the null preference differences $\left(w_{i}, w_{i}\right)$ always belong to the first equivalence class of $\succsim_{i}^{*}$.

As discussed in Bouyssou and Pirlot (2005a, Sect. 5.2), the result of Greco et al. (2001a) on concordance relations deals with a particular class of R-CR, in contrast with our own results. Furthermore, as this analysis does not explicitly uses conditions $R C 1$ and $R C 2$, it is not conducted within the broader framework of model (M). Since, in our view, the rôle of axiomatization is mainly to give a sound basis for the comparison of several preference models, we tend to view this point as an advantage of our approach (our view on axiomatization is developed in Bouyssou and Pirlot 2005a, section 5.3).

Greco et al. (2001a) have noted that adding conditions $A C 1, A C 2$ and $A C 3$ implies that the resulting relations $S_{i}$ must be semiorders. Nevertheless, they have not studied the independence of these conditions with respect to conditions (23) and (24). As shown in Bouyssou and Pirlot (2005a, Lemma 27), conditions $A C 1_{i}$ and $A C 2_{i}$ turn out to be equivalent for $\mathrm{R}$-CR. Independence issues were indeed the most delicate ones to tackle in Bouyssou and Pirlot (2005a, 2007b). 
Although we already mentioned in Bouyssou et al. (1997) that model (M) offers an adequate framework to tackle R-CDR, it took us some time to devise adequate conditions that would characterize R-CDR within this framework. Greco et al. (2001a) were the first to come up with such conditions. The main condition they use to characterize R-CDR satisfying (22) is the following. We say that $\succsim$ is super-coarse with veto on attribute $i \in N$ if, for all $x_{i}, y_{i}, z_{i}, w_{i}, r_{i}, s_{i} \in X_{i}$ and all $a_{-i}, b_{-i}, c_{-i}, d_{-i} \in X_{-i}$,

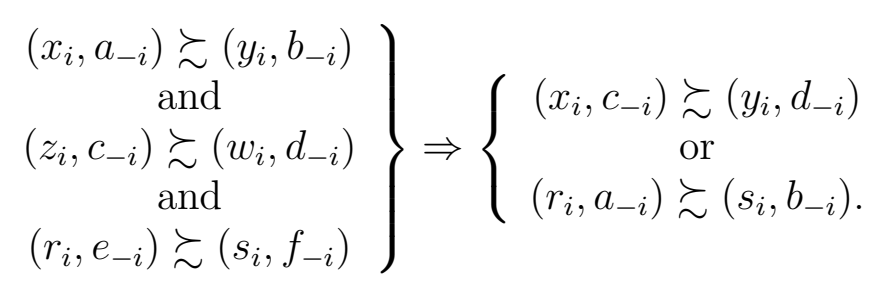

This condition is a clear weakening of (23) that has inspired our own weakening of $M 2$ to obtain M3. The main result in Greco et al. (2001a) is that R-CDR satisfying (22) are characterized by the conjunction of conditions (24) and (25). We do not repeat here the comments made above: this result is not conducted within a broader framework, like the one provided by model (M), and only deals with a particular class of R-CDR. Nevertheless, it was the first approach that convincingly took discordance into account in a conjoint measurement setting.

\section{References}

Bouyssou, D., 1986. Some remarks on the notion of compensation in MCDM. European Journal of Operational Research 26, 150-160.

Bouyssou, D., 1992. On some properties of outranking relations based on a concordance-discordance principle. In: Duckstein, L., Goicoechea, A., Zionts, S. (Eds.), Multiple criteria decision making. Springer-Verlag, Berlin, pp. 93-106.

Bouyssou, D., 1996. Outranking relations: Do they have special properties? Journal of Multi-Criteria Decision Analysis 5, 99-111.

Bouyssou, D., Marchant, Th., 2007a. An axiomatic approach to noncompensatory sorting methods in MCDM, I: The case of two categories. European Journal of Operational Research 178 (1), 217-245.

Bouyssou, D., Marchant, Th., 2007b. An axiomatic approach to noncompensatory sorting methods in MCDM, II: More than two categories. European Journal of Operational Research 178 (1), 246-276.

Bouyssou, D., Marchant, Th., Pirlot, M., Tsoukiàs, A., Vincke, Ph., 2006. Evaluation and decision models: Stepping stones for the analyst. Springer, New York. 
Bouyssou, D., Pirlot, M., 1999. Conjoint measurement without additivity and transitivity. In: Meskens, N., Roubens, M. (Eds.), Advances in Decision Analysis. Kluwer, Dordrecht, pp. 13-29.

Bouyssou, D., Pirlot, M., 2002a. A characterization of strict concordance relations. In: Bouyssou, D., et al. (Eds.), Aiding Decisions with Multiple Criteria: Essays in Honour of Bernard Roy. Kluwer, Dordrecht, pp. 121-145.

Bouyssou, D., Pirlot, M., 2002b. Nontransitive decomposable conjoint measurement. Journal of Mathematical Psychology 46 (6), 677-703.

Bouyssou, D., Pirlot, M., 2004a. 'Additive difference' models without additivity and subtractivity. Journal of Mathematical Psychology 48 (4), 263-291.

Bouyssou, D., Pirlot, M., 2004b. Preferences for multiattributed alternatives: Traces, dominance, and numerical representations. Journal of Mathematical Psychology 48 (3), 167-185.

Bouyssou, D., Pirlot, M., 2005a. A characterization of concordance relations. European Journal of Operational Research 167 (2), 427-443.

Bouyssou, D., Pirlot, M., 2005b. Following the traces: An introduction to conjoint measurement without transitivity and additivity. European Journal of Operational Research 163 (2), 287-337.

Bouyssou, D., Pirlot, M., 2007a. An axiomatic analysis of concordance-discordance relations. Cahiers du LAMSADE 269, LAMSADE, Université Paris Dauphine, Also available at http://lamsade.dauphine.fr/ bouyssou.

Bouyssou, D., Pirlot, M., 2007b. Further results on concordance relations. European Journal of Operational Research 181 (1), 505-514.

Bouyssou, D., Pirlot, M., Vincke, Ph., 1997. A general model of preference aggregation. In: Karwan, M. H., Spronk, J., Wallenius, J. (Eds.), Essays in Decision Making. Springer Verlag, Berlin, pp. 120-134.

Bouyssou, D., Vansnick, J.-C., 1986. Noncompensatory and generalized noncompensatory preference structures. Theory and Decision 21, 251-266.

Brans, J.-P., Mareschal, B., 2002. PROMETHEE-GAIA. Une méthodologie d'aide à la décision en présence de critères multiples. Éditions de l'Université de Bruxelles, Brussels. 
Doignon, J.-P., Monjardet, B., Roubens, M., Vincke, Ph., 1988. Biorder families, valued relations and preference modelling. Journal of Mathematical Psychology $30,435-480$.

Dubois, D., Fargier, H., Perny, P., Prade, H., 2003. A characterization of generalized concordance rules in multicriteria decision-making. International Journal of Intelligent Systems 18 (7), 751-774.

Fishburn, P. C., 1975. Axioms for lexicographic preferences. Review of Economic Studies 42, 415-419.

Fishburn, P. C., 1976. Noncompensatory preferences. Synthese 33, 393-403.

Greco, S., Matarazzo, B., Słowiński, R., 2001a. Axiomatic basis of noncompensatory preferences, communication to $F U R X, 30$ May-2 June, Torino, Italy.

Greco, S., Matarazzo, B., Słowiński, R., 2001b. Rough sets theory for multicriteria decision analysis. European Journal of Operational Research 129 (1), 1-47.

Greco, S., Matarazzo, B., Słowiński, R., 2005. Decision rule approach. In: Figueira, J., Greco, S., Ehrgott, M. (Eds.), Multiple Criteria Decision Analysis: State of the Art Surveys. Springer-Verlag, Boston, pp. 507-562.

Keeney, R. L., Raiffa, H., 1976. Decisions with multiple objectives: Preferences and value tradeoffs. Wiley, New-York.

Krantz, D. H., Luce, R. D., Suppes, P., Tversky, A., 1971. Foundations of measurement. Vol. 1: Additive and polynomial representations. Academic Press, New-York.

Luce, R. D., 1956. Semiorders and a theory of utility discrimination. Econometrica 24, 178-191.

Pirlot, M., 1997. A common framework for describing some outranking procedures. Journal of Multi-Criteria Decision Analysis 6, 86-92.

Roy, B., 1968. Classement et choix en présence de points de vue multiples (la méthode ELECTRE). RIRO 2, 57-75.

Roy, B., 1978. ELECTRE III : un algorithme de classement fondé sur une représentation floue des préférences en présence de critères multiples. Cahiers du CERO 20, 3-24.

Roy, B., 1991. The outranking approach and the foundations of ELECTRE methods. Theory and Decision 31, 49-73. 
Roy, B., Bertier, P., 1973. La méthode ELECTRE II : une application au mediaplanning. In: Ross, M. (Ed.), OR'72. North Holland, Amsterdam, pp. 291-302.

Roy, B., Bouyssou, D., 1993. Aide multicritère à la décision : méthodes et cas. Economica, Paris.

Tsoukiàs, A., Perny, P., Vincke, Ph., 2002. From concordance / discordance to the modelling of positive and negative reasons in decision aiding. In: Bouyssou, D., et al. (Eds.), Aiding Decisions with Multiple Criteria: Essays in Honour of, Bernard Roy. Kluwer, Dordrecht, pp. 147-174.

Tversky, A., 1969. Intransitivity of preferences. Psychological Review 76, 31-48.

Vansnick, J.-C., 1986. On the problems of weights in MCDM (the noncompensatory approach). European Journal of Operational Research 24, 288-294. 